\title{
Molecular cloning and expression analysis of the first two key genes through 2-C-methyl-D-erythritol 4-phosphate (MEP) pathway from Pyropia haitanensis (Bangiales, Rhodophyta)
}

\author{
Yu Du ${ }^{1}$, Jian Guan ${ }^{1}$, Ruijun $\mathrm{Xu}^{2}$, Xin Liu ${ }^{1}$, Weijie Shen ${ }^{1}$, Yafeng $\mathrm{Ma}^{1}$, Yuan $\mathrm{He}^{1}$, Songdong \\ Shen ${ }^{1, *}$
}

${ }^{1}$ Department of Cell Biology, School of Biology and Basic Medical, Soochow University, No. 199 Renai Road, Suzhou 215123, China

${ }^{2}$ School for Radiological \& Interdisciplinary Sciences, Soochow University, No. 199 Renai Road, Suzhou 215123, China

\begin{abstract}
Pyropia haitanensis (T. J. Chang et B. F. Zheng) N. Kikuchi et M. Miyata is one of the most commercially useful macroalgae cultivated in southeastern China. In red algae, the biosynthesis of terpenoids through 2-C-methyl-Derythritol 4-phosphate (MEP) pathway can produce a direct influence on the synthesis of many biologically important metabolites. In this study, two genes of cDNAs, 1-deoxy-D-xylulose-5-phosphate synthase (DXS) and 1-deoxy-Dxylulose-5-phosphate reductase (DXR), which encoding the first two rate-limiting enzymes among MEP pathway were cloned from P. haitanensis. The cDNAs of P. haitanensis DXS (PhDXS) and DXR (PhDXR) both contained complete open reading frames encoding polypeptides of 764 and 426 amino acids residues, separately. The expression analysis showed that PhDXS was significant differently expressed between leafy thallus and conchocelis as $P h D X R$ been non-significant. Additionally, expression of PhDXR and its downstream gene geranylgeranyl diphosphate synthase were both inhibited by fosmidomycin significantly. Meanwhile, we constructed types of phylogenetic trees through different algae and higher plants DXS and DXR encoding amino acid sequences, as a result we found tree clustering consequences basically in line with the "Cavalier-Smith endosymbiotic theory." Whereupon, we speculated that in red algae, there existed only complete MEP pathway to meet needs of terpenoids synthesis for themselves; Terpenoids synthesis of red algae derivatives through mevalonate pathway came from two or more times endosymbiosis of heterotrophic eukaryotic parasitifer. This study demonstrated that PhDXS and PhDXR could play significant roles in terpenoids biosynthesis at molecular levels. Meanwhile, as nuclear genes among MEP pathway, PhDXS and PhDXR could provide a new way of thinking to research the problem of chromalveolata biological evolution.
\end{abstract}

Key Words: gene expression analysis; MEP pathway; molecular cloning; Pyropia haitanensis

Abbreviations: AOX, alternative oxidase; DMAPP, dimethylallyl diphosphate; DXP, 1-deoxy-D-xylulose-5-phosphate; DXR, 1-deoxy-D-xylulose-5-phosphate reductase; DXS, 1-deoxy-D-xylulose-5-phosphate synthase; GGPS, geranylgeranyl diphosphate synthase; IPP, isopentenyl diphosphate; MEP, 2-C-methyl-D-erythritol 4-phosphate; MVA, mevalonate; ORF, open reading frame; PCR, polymerase chain reaction; PhDXS, Pyropia haitanensis DXS; PhDXR, Pyropia haitanensis DXR; RT-PCR, reverse transcription polymerase chain reaction; SRA, sequence read archive; UTR, untranslated region

(1) \$ This is an Open Access article distributed under the terms of the Creative Commons Attribution Non-Commercial License (http://creativecommons.org/licenses/by-nc/3.0/) which permits unrestricted non-commercial use, distribution, and reproduction in any medium, provided the original work is properly cited.
Received August 27, 2017, Accepted October 2, 2017

*Corresponding Author

E-mail: shensongdong@suda.edu.cn

Tel: +86-513-65880276, Fax: +86-512-511-2065 


\section{INTRODUCTION}

Pyropia haitanensis (T. J. Chang et B. F. Zheng) N. Kikuchi et M. Miyata, as a typical species of Bangiales growing in intertidal zones on the coast, is an important marine crop with huge economic benefits (Luo et al. 2014). Similar to other genus Pyropia of more than 75 species (Sutherland et al. 2011), P. haitanensis has an alternation of heteromorphic generations (Blouin et al. 2011, Yang et al. 2013, Luo et al. 2014, Chen et al. 2016), with a leafy thallus phase (gametophyte) and a filamentous conchocelis phase (sporophyte). Compared with other Pyropia species (mainly Porphyra yezoensis), its annual output is higher and the cropped location is more single: only along the coasts of South China (Chan et al. 2012). And more importantly, as a kind of oceanic green food, it can be made for nori, and serving as ingredients of soup and sushi, which is well loved by the public all over the world.

As we know, terpenoids, such as tocopherol, gibberellins, carotenoids, and chlorophyll, are the main biological components in Pyropia, and the efficiency of terpenoid synthesis will directly affect the yield of Pyropia (Yang et al. 2016). The biosynthesis of terpenoids in plants, is to generate two common precursors, isopentenyl diphosphate (IPP) and dimethylallyl diphosphate (DMAPP) through the mevalonate (MVA) pathway and the methylerythritol phosphate (MEP) pathway (Tong et al. 2015). However, MEP pathway is the only terpenoid biosynthetic pathway in lower algal organisms (Massé et al. 2004, Pattanaik and Lindberg 2015). Fig. 1 summarizes the series of reactions, genes and enzymes among the MEP pathway as identified in Pyropia (Chan et al. 2012). Within MEP pathway, the first gene 1-deoxy-D-xylulose-5-phosphate synthase (DXS) catalyzes the conversion from pyruvate and D-glyceraldehyde-3-phosphate to 1-deoxy-D-xylulose-5-phosphate (DXP), and the second gene 1-deoxyD-xylulose-5-phosphate reductase (DXR) catalyzes DXP to generate 2-C-methyl-D-erythritol 4-phosphate (MEP). Both of the two enzymes are accounted as potential regulators of carbon flux among the MEP pathway (Tong et al. 2015). A multitude of studies suggest that overexpression of DXS can lead to elevated levels of terpenoids (Liu et al. 2015), while overexpression of DXR shows an increased accumulation of terpenoids such as carotenoids and chlorophyll (Carretero-Paulet et al. 2006). In particular, a specific inhibitor of DXR, fosmidomycin, when it was used to treat mature plants such as tomatoes and green algae, accumulation of terpenoids would be inhibited (Kuzuyama et al. 1998, Rodríguez-Concepción et al.

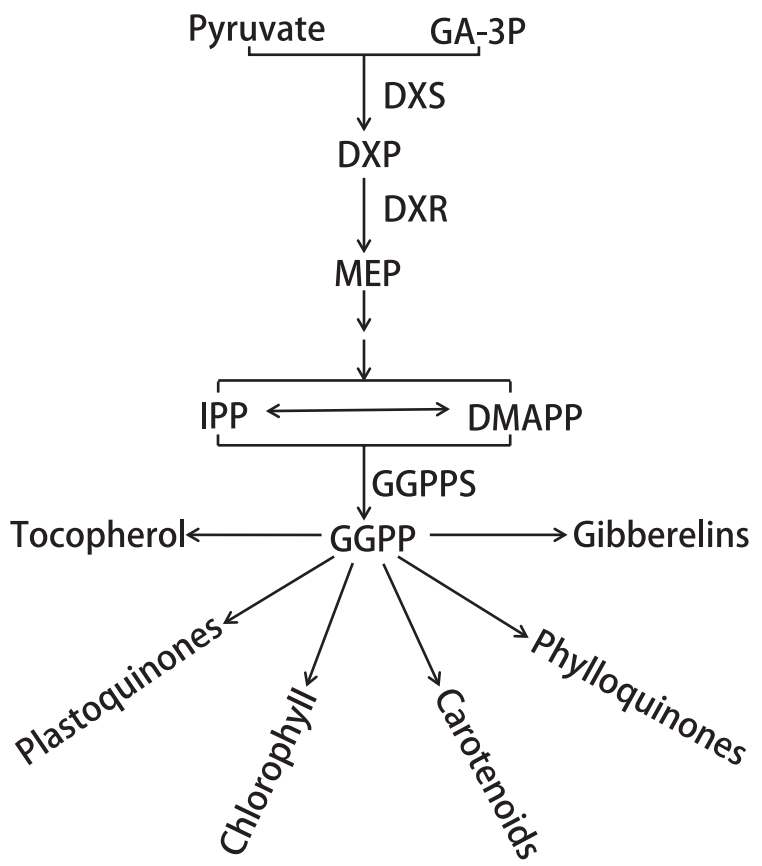

Fig. 1. The MEP pathway for isoprenoid biosynthesis in Pyropia. GA-3P, glyceraldehyde-3-phosphate; DXS, 1-deoxy-D-xylulose 5-phosphate synthase; DXP, 1-deoxy-D-xylulose 5-phosphate; DXR, 1-deoxy-D-xylulose 5-phosphate reductoisomerase; MEP, 2-C-methylD-erythritol 4-phosphate; IPP, isopentenyl diphosphate; DMAPP, dimethylallyl diphosphate; GGPPS, geranylgeranyl diphosphate synthase; GGPP, geranylgeranyl diphosphate.

2001). In addition, in the downstream of the terpenoid synthesis, there is also a geranylgeranyl diphosphate synthase (GGPS) gene, which can catalyze geranylgeranyl diphosphate, a metabolic intermediate in the biosynthesis of terpenoids (Scolnik and Bartley 1994). Previous studies suggest that the expression level of downstream GGPS can be induced by upstream genes among the MEP pathway in plants and are accordant in expression between them both (Okada et al. 2000).

Certain bacteria, some protozoa and animals merely use the MVA pathway (Massé et al. 2004), while not only photosynthetic autotrophs such as red algae, green algae and higher plants require the plastidic MEP pathway to synthesize terpenes for their metabolic needs, but also some of the non-photosynthetic heterophytes such as Crypthecodinium cohnii, Oxyrrhis marina, and Perkinsus marinus require the MEP pathway for terpenoid synthesis (Slamovits and Keeling 2008). There are seven enzymes involved in the MEP pathway, all of which are encoded by the nuclear gene. And then the enzymes locate in the cytoplasm under the action of the transit peptide to exert the corresponding activity (Vranová et al. 2013). It 

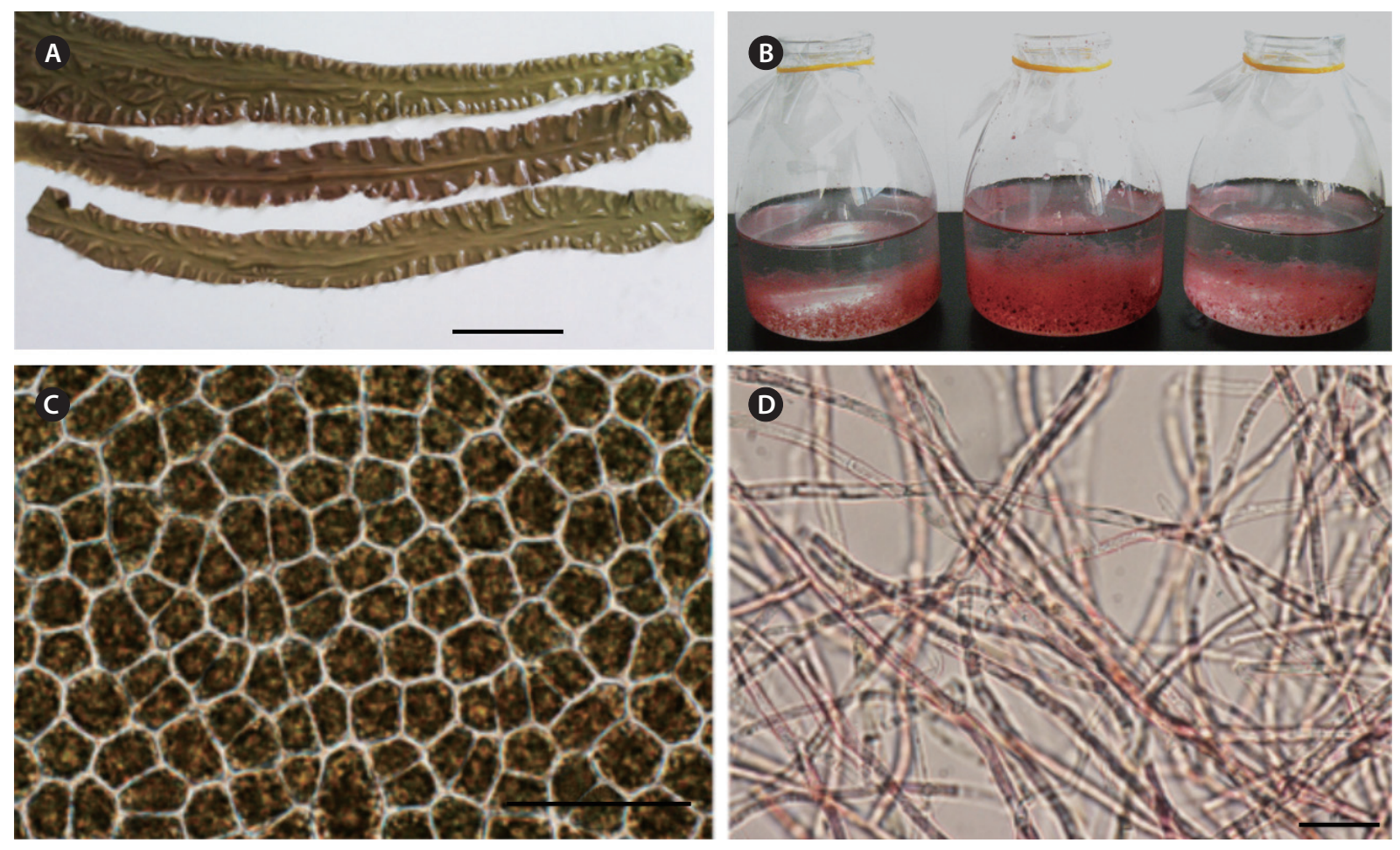

Fig. 2. The growth of leafy thallus and filamentous thallus from Pyropia haitanensis at $18^{\circ} \mathrm{C}$. (A \& C) The leafy thallus and its microscopic picture. (B \&D) The filamentous thallus in bottles and its microscopic picture. Scale bars represent: $A, 3 \mathrm{~cm} ; C$ \&, $25 \mu \mathrm{m}$.

is useful of MEP pathway which provides a new direction for the study of biological system evolution.

As for P. haitanensis in chromalveolata, it is considered that through the primary endosymbiosis, Cyanophyta evolved into three major categories of existing algae, namely red algae, green algae, and glaucophyte. Red algae through a series of secondary endosymbiosis, become the only common ancestor of many algae such as haptopflagellates, stramenopiles, dinoflagellates, and cryptophytes (Cavalier-Smith 1999). In this study, we hope to use key genes DXS and DXR in the MEP pathway to construct the phylogenetic tree for analyzing the evolutionary relationship between different algae in chromalveolata and higher terrestrial plants, in addition, for analyzing the possibility of MVA pathway existing in red algae and the endosymbiotic sources of red algae derivative in the MVA terpenoid synthesis pathway. Thus, a first step in this study would be to clone and characterize the DXS and DXR genes from P. haitanensis. Moreover, expression levels of $P$. haitanensis DXS (PhDXS) and DXR $(P h D X R)$ in different life phases were analyzed, and expression analysis of PhDXR and PhGGPS in different fosmidomycin concentrations were investigated.

\section{MATERIALS AND METHODS}

The leafy thallus of $P$. haitanensis was collected from Putian, Fujian province in China and the same strain of conchocelis were purchased from Jiangsu Research Institute of Laver. Meanwhile, by utilizing techniques in separation and purification, $P$. haitanensis could be obtained with no miscellaneous algae pollution and then cultivated in the laboratory from the Department of Cell Biology, Soochow University (Fig. 2A \& B). The distinct condition for cultivation of the leafy thallus was $15 \mathrm{mg}$ $\mathrm{L}^{-1} \mathrm{~N}$ eutrophic brine as the conchocelis be $2 \mathrm{mg} \mathrm{L}^{-1} \mathrm{~N}$ eutrophic brine. Anything else, both of them were grown and maintained at $18^{\circ} \mathrm{C}$, and at a light intensity of $50 \mu \mathrm{mol}$ photons $\mathrm{m}^{-2} \mathrm{~s}^{-1}$ with a $12: 12 \mathrm{~h}$ photoperiod (Blouin et al. 2011, Chen et al. 2016).

\section{RNA and DNA isolation}

Total RNA was extracted from leafy thallus and conchocelis of $P$. haitanensis with cryoperservation, using a MiniBEST Plant RNA Extraction Kit (TaKaRa, Tokyo, Japan), according to steps in the instruction manual. Afterwards, samples were dissolved in diethylpyrocarbonate-treated 
water and digested with DNaseI so as to obtain RNA of high levels. The genomic DNA was isolated from P. haitanensis according to a previously described cetyltrimethylammonium bromide methods (Yang et al. 2013). The concentration and quality of the extracted DNA and RNA were preliminary tested by electrophoresis on $1 \%$ agarose gels. DNA stripes with bright and single while RNA ones with three bright bands were stored at $-70^{\circ} \mathrm{C}$ until used.

\section{Molecular cloning of cDNA of PhDXS and PhGGPS}

The cDNA sample was reverse-transcribed from $2 \mu \mathrm{g}$ of total RNA with $500 \mathrm{ng}$ oligo (dT) primers according to manufacturer's instructions (TaKaRa). The cDNA was used as a masterplate for amplification of unknown fragments of PhDXS and PhGGPS. According to analyze genome and transcriptome from similar species of $P$. umbilicalis and $P$. purpurea, a set of gene-specific primers DXSCF, DXSCR, GGPSF, and GGPSR were designed for reverse transcription-polymerase chain reaction (RTPCR) (Table 1). The systems were both carried out in a total volume of $50 \mu \mathrm{L}$ containing $39.5 \mu \mathrm{LddH}_{2} \mathrm{O}, 2 \mu \mathrm{L} 10 \times$ PCR Buffer II ( $\mathrm{Mg}^{2+}$ plus), $2 \mu \mathrm{L}$ dNTP mixture $(10 \mathrm{mM})$, $2 \mu \mathrm{L}$ cDNA, $1 \mu \mathrm{L}$ forward primer $(20 \mu \mathrm{M}), 1 \mu \mathrm{L}$ reverse primer $(20 \mu \mathrm{M})$, and $0.5 \mu \mathrm{L}$ TaKaRa Ex Taq HS (5 U/ $\mu \mathrm{L}$; TaKaRa). Next, the PCR products were amplified and cloned into the pEASY-T3 vector (TransGen, Beijing, China) and sequenced using Genewiz Biological Technology Co., Ltd (Suzhou, China). The obtained sequences were compared to other plants of DXS and GGPS genes in the NCBI database from nucleotide BLAST to identify the most similar sequences exact genes that we need. In addition, to validate if there were introns in the core fragments of $P h D X S$ and $P h G G P S$, the leafy thallus genomic DNA was used as a template for general PCR. The gene-specific primers DXSCF, DXSCR, GGPSF, and GGPSR were used to amplify products (Table 1). The two systems were performed in a total volume of $50 \mu \mathrm{L}$ containing $35.5 \mu \mathrm{L}$ $\mathrm{ddH}_{2} \mathrm{O}, 5 \mu \mathrm{L}$ 10× LA Taq PCR Buffer II ( $\mathrm{Mg}^{2+}$ plus), $5 \mu \mathrm{L}$ dNTP mixture $(2.5 \mathrm{mM}), 2 \mu \mathrm{L}$ genomic DNA, $1 \mu \mathrm{L}$ forward / reverse primer $(20 \mu \mathrm{M})$, and $0.5 \mu \mathrm{L}$ TaKaRa LA Taq (5 U/ $\mu \mathrm{L})$. Afterwards, sequences between RT-PCRs and PCRs were compared and analyzed with DNAMAN and NCBI BLAST. The core sequence of $P h D X S$ was used to design 5 '-RACE primers.

The 5'-ready cDNA was obtained from $2 \mu \mathrm{L}$ reversetranscribed total RNA with 5'-CDS PrimerA. The 5'-RACE Outer PCR primer DXSGspR1 and Inner PCR primer

Table 1. The primers used in the present study of Pyropia haitanensis

\begin{tabular}{|c|c|c|c|}
\hline Gene & Primer name & Sequence $\left(5^{\prime}-3^{\prime}\right)$ & Product size (bp) \\
\hline \multirow{10}{*}{ DXS } & Core fragment & - & 2,363 \\
\hline & $\mathrm{CF}$ & GACCGTCGCCCTCCACTTTG & - \\
\hline & $\mathrm{CR}$ & CCACGACGATTGGTCAGAAA & - \\
\hline & 5' RACE & - & 857 \\
\hline & Long primer & CTAATACGACTCACTATAGGGCAAGCAGTGGTATCAACGCAGAGT & - \\
\hline & Short primer & CTAATACGACTCACTATAGGGC & - \\
\hline & GspR1 & GGACGTCGGAAAAGGGCTTGGA & - \\
\hline & GspR2 & TCGTATGCCATGCCACCCGTAA & - \\
\hline & $\mathrm{YZ}$ & - & 1,193 \\
\hline & YZF & ATCCCACCTCCGTATTGCAT & - \\
\hline \multirow{7}{*}{ DXR } & YZR & GTCCAGGTTGTGCCCGTCAATG & - \\
\hline & DXR mRNA & - & 1,474 \\
\hline & F1 & GCCATCGCCTTCCCGCTTCG & - \\
\hline & $\mathrm{R} 1$ & TGTGCAGCAGCCCTGTCATA & - \\
\hline & DXR DNA & - & 1,458 \\
\hline & $\mathrm{F} 2$ & TCCACCCGCCATCGCCTTCC & - \\
\hline & $\mathrm{R} 2$ & AGCAGAAAACGACGGACATG & - \\
\hline \multirow{3}{*}{ GGPS } & Partial fragment & - & 612 \\
\hline & $\mathrm{F}$ & TCGCTCATCCACGACGACCTCC & - \\
\hline & $\mathrm{R}$ & CGCCGCCTTGTCGCCATACA & - \\
\hline \multirow[t]{2}{*}{ q18SrRNA } & $\mathrm{F}$ & GATCGAAGACGATCAGATACCG & 210 \\
\hline & $\mathrm{R}$ & GTTGAGTCAAATTAAGCCGCAG & - \\
\hline \multirow[t]{2}{*}{ qDXR } & $\mathrm{F}$ & CCGTGCATTGAGCTGGCCTAT & 246 \\
\hline & $\mathrm{R}$ & AGCATCCACAAACCCATTTACCA & - \\
\hline \multirow[t]{2}{*}{ qGGPS } & $\mathrm{F}$ & AGTGATGGACCTGGAGAGTGA & 259 \\
\hline & $\mathrm{R}$ & GTCCTTGCCCGACGTTTTG & - \\
\hline
\end{tabular}

DXS, 1-deoxy-D-xylulose-5-phosphate synthase; DXR, 1-deoxy-D-xylulose-5-phosphate reductase; GGPS, geranylgeranyl diphosphate synthase. 
DXSGspR2 (Table 1), were designed on the basis of the cloned core fragments of $P h D X S$. The 5'-RACE was carried out using SMARTer RACE 5'/3' Kit User Manual Extraction Kit (TaKaRa) as instructions. Then, amplified 5'-RACE fragments were purified and cloned into the pEASY-T3 vector (TransGen) followed by sequencing. Meanwhile, to test if there were any introns in the complete sequence of PhDXS, the cDNA was used as a template for RT-PCR. The $5^{\prime}$ region designed primer DXSYZF and the middle position of core fragment designed primer DXSYZR were used to amplify products (Table 1). Finally, the complete open reading frame (ORF) of PhDXS was obtained and submitted to the GenBank database.

\section{Cloning of a complete ORF of PhDXR}

Since we analyzed and designed gene-specific primers of $P h D X R$ according to conservative regions of amino and nucleic acids from numerous similar species. The Pyropia umbilicalis DXR (PUDXR) 5' untranslated region (5' UTR) designed primer DXRF1 and the PUDXR 3' UTR designed primer DXRR1 according to transcriptome of P. umbilicalis DXR were used to amplify products. The above-mentioned cDNA was used as a template. Fortunately, the complete ORF of PhDXR was obtained and revalidated through a pair of primers DXRF2 and DXRR2.

\section{Bioinformatic analysis of $P h D X S$ and $P h D X R$}

DNAMAN software and NCBI BLAST (http://blast. ncbi.nlm.nih.gov/Blast.cgi) were used to assemble the obtained sequences into the cDNA of PhDXS and PhDXR gene. The deduced amino acid sequences were analyzed using NCBI ORF Finder (https://www.ncbi.nlm.nih.gov/ orffinder/) and the Conserved Domain Search. The encoding protein domains were predicted by NCBI blastx and blastn. TargetP 1.1 Server (http://www.cbs.dtu.dk/ services/TargetP/), SignalP 4.1 Server (http://www.cbs. dtu.dk/services/SignalP/), and ChloroP 1.1 Server (http:// www.cbs.dtu.dk/services/ChloroP/) predicted the subcellular localization, the potential signal peptide and the transit peptide. Secondary structure of proteins were predicted by NPS@ : SOPMA.

The neighbor joining phylogenetic tree for $P h D X R$ gene and DXS proteins was generated using the statistical maximal parsimony method with default parameters of 1,000 bootstrap replications. Meanwhile, phylogenetic tree for PhDXS gene was established with maximum likelihood method. Sequences of all used species used in the phylogenetic tree were downloaded from the
NCBI database. Amino acid sequences were aligned using the CLUSTAL W program, and phylogenetic trees using MEGA5 software by poisson model. Bootstrap values obtained after 1,000 replications are shown on the branches.

\section{Expression analysis of PhDXS and PhDXR in dif- ferent generation periods}

Semiquantitative RT-PCR was used to explore the expression patterns of $P h D X S$ and $P h D X R$ in different life history of leafy thallus and conchocelis. Gene-specific primers qDXSF, qDXSR for $P h D X S$ and qDXRF, qDXRR for PhDXR were used (Table 1). Primers for house-keeping gene 18S rRNA (q18SrRNAF and q18SrRNAR) were designed as an internal control. The amplified products were separated on $1 \%$ agarose and photographed on Vilber Lourmat (Bio-RAD, Hercules, CA, USA). In addition, quantitative real-time PCR was used to investigate it deeply. For real-time PCR, pairs of gene-specific primers of DXS, DXR and actin gene 18S rRNA were the same as above. PCR products were then quantified constantly with ABI7500 qPCR System (Applied Biosystems, Foster City, CA, USA) using SYBR green fluorescence (TaKaRa) according to the instructions. Through 2 thermal cycler, the system was under the following conditions: $95^{\circ} \mathrm{C}$ for $30 \mathrm{~s}$, followed by 40 cycles of amplification $\left(95^{\circ} \mathrm{C}\right.$ for $5 \mathrm{~s}$, $60^{\circ} \mathrm{C}$ for $\left.34 \mathrm{~s}\right)$.

\section{Expression analysis of downstream PhGGPS ef- fected by MEP pathway}

Fosmidomycin (Absin Bioscience lnc., Shanghai, China), as a natural antibiotic, which can inhibit DXR in the MEP pathway for terpenoids biosynthesis (Kuzuyama et al. 1998), were added to sodium salt brine. For fosmidomycin treatment, the tissues were cultivated with three different concentrations: 0,200 , and $300 \mu \mathrm{M} \mathrm{L}^{-1}$ for $72 \mathrm{~h}$. Each concentration was performed in triplicate. First, to explore the effect of fosmidomycin in the process of MEP pathway of $P$. haitanensis, extraction of terpenoid biosynthetic end-products such as chlorophyll and phycobiliproteins from leafy thallus were needed. After centrifugation for $15 \mathrm{~min}$ at $4^{\circ} \mathrm{C} 12,000 \mathrm{rpm}$, the chlorophyll, C-phycocyanin, allophycocyanin, and phycobiliproteins were extracted in the supernatant. Calculation methods of phycobiliproteins referenced to Bennett and Bogorad (1973), and chlorophyll were calculated following the way of Wellburn (1994). The concentrations of chlorophyll and phycobiliproteins were measured by Synergy 


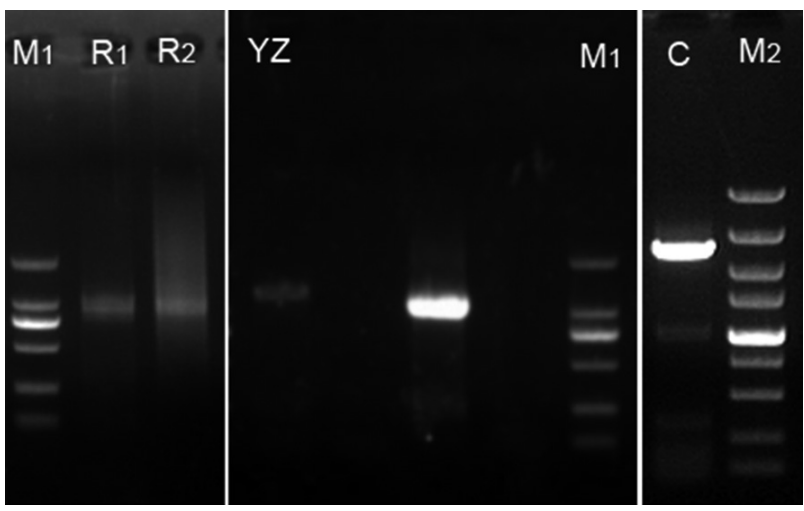

Fig. 3. The PCR products of DXS CDNA gene from Pyropia haitanensis. M1, Takara DL2000 bp DNA marker; R1, 5' RACE PCR: 857 bp; R2, 5' RACE Nest PCR: 857 bp; YZ, checking fragment: 1,027 bp; C, DXS core fragment: 2,363 bp; M2, Takara DL5000 bp DNA marker. PCR, polymerase chain reaction; DXS, 1-deoxy-D-xylulose 5-phosphate synthase.
A

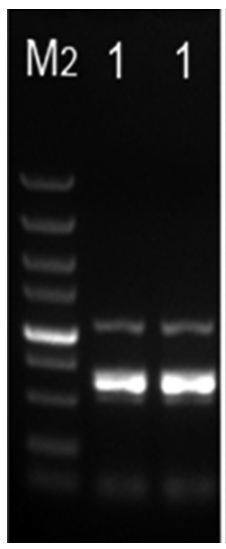

B

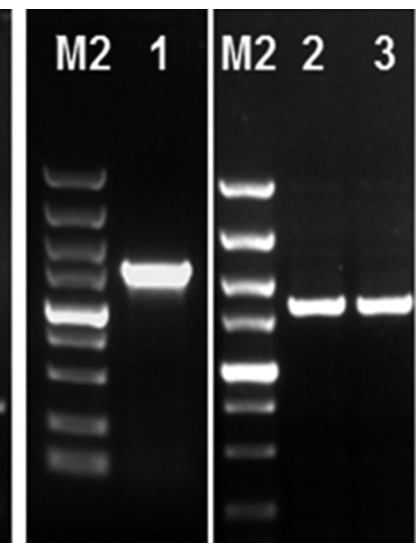

Fig. 4. PCR products of GGPS and DXR genes. (A) The partial GGPS from Pyropia haitanensis. M2, Takara DL5000 bp DNA marker; 1, GGPS DNA gene: 612 bp; 2, GGPS cDNA gene: 612 bp. (B) DXR from Pyropia haitanensis. M2, Takara DL5000 bp DNA marker; 1, DXR DNA gene: 1,458 bp; 2 \& 3, DXR cDNA gene: 1,474 bp. PCR, polymerase chain reaction; GGPS, gene geranylgeranyl diphosphate synthase; DXR, 1-deoxy-D-xylulose 5-phosphate reductoisomerase.

(Biotek, Winooski, VT, USA) between absorption spectra of 470 and $665.2 \mathrm{~nm}$. And then, in order to explore the effect of fosmidomycin inhibitor on DXR and downstream GGPS effected by MEP pathway at the molecular level, quantitative real-time PCR was used above-mentioned. Rather than using leafy thallus, conchocelis used in this study (Xu et al. 2012) were collected from 1-month-old $P$. haitanensis, continuous fresh culturing until used. Besides, a pair of primers designed for GGPS (qGGPSF and qGGPSR) were simultaneously used.

\section{Statistical analyses}

Expression data of $P$. haitanensis DXS and DXR genes in different growth periods would use $2^{-\Delta \Delta C t}$ relative quantitative method (Livak method) to analysis. To explore the gene expression of DXS relative to DXR, we might firstly use different life cycles of DXS and DXR for normalized processing with internal standard gene 18 SrRNA, then the leafy thallus and conchocelis adopted formula $2^{-\Delta(C t D X R-C t}$ internal standard) $/ 2^{-\Delta(C t D X S C t}$ internal standard) to calculate gene relative expression. Next, Origin 8.0 software (Origin Lab, Northampton, MA, USA) was used to perform Oneway analysis of variance (ANOVA) and Scheffé post-mortem examination, the significance level was set at alpha $=0.01 / 0.05$.

\section{RESULTS}

\section{Cloning and characterization of PhDXS and PhDXR}

According to the comparative analysis of DXS transcripts of P. umbilicalis and P. purpurea (P_umbilicalis_esi sotig02238, P_purpurea_esGAQG33Y02G5QZS) and DXS of different species, the DXSCF / DXSCR primers were designed in the amino acid and nucleic acid conserved region. A 2,363 bp fragment was obtained by RT-PCR (Fig. 3C). By using 5'-RACE technology on the basis of the core fragments, the sequence of $857 \mathrm{bp} P h D X S$ cDNA was obtained and analyzed (lanes R1 and R2 in Fig. 3). At the same time, according to the comparative analysis of DXR transcripts of $P$. umbilicalis and P. purpurea $\left(\mathrm{P}_{-}\right.$ umbilicalis_esContig5003, P_purpurea_esi sotig05945, and P_purpurea_ esGDOFQBI01DQXCK) and DXR of different species, the cDNA sequence of $P h D X R$ with 1,474 bp was acquired via a pair of primers DXRF1 and DXRR1 by RT-PCR (lanes 2 and 3 in Fig. 4B). Through general PCR of genomic DNA, the results showed that the checking fragments of DXS designed by primers DXSYZF / DXSYZR was 1,027 bp (lane YZ in Fig. 3), and the sequence of DXR DNA gene designed by primers DXRF2 / DXRR2 was 1,458 bp (lane 1 in Fig. 4B), two genes both contained no intron. Through the final splicing and comparison analysis, we confirmed that the cDNA of PhDXS was 2,911 bp (Genbank accession No. KY697780) and contained an open reading frame of 2,295 bp including $102 \mathrm{bp}$ of 5 ' UTR and 514 bp of $3^{\prime}$ UTR. PhDXR gene with a length of 1,474 bp (Genbank accession No. KY697779) possessed 1,281 bp open reading frame with two flanks of $21 \mathrm{bp}$ and 172 

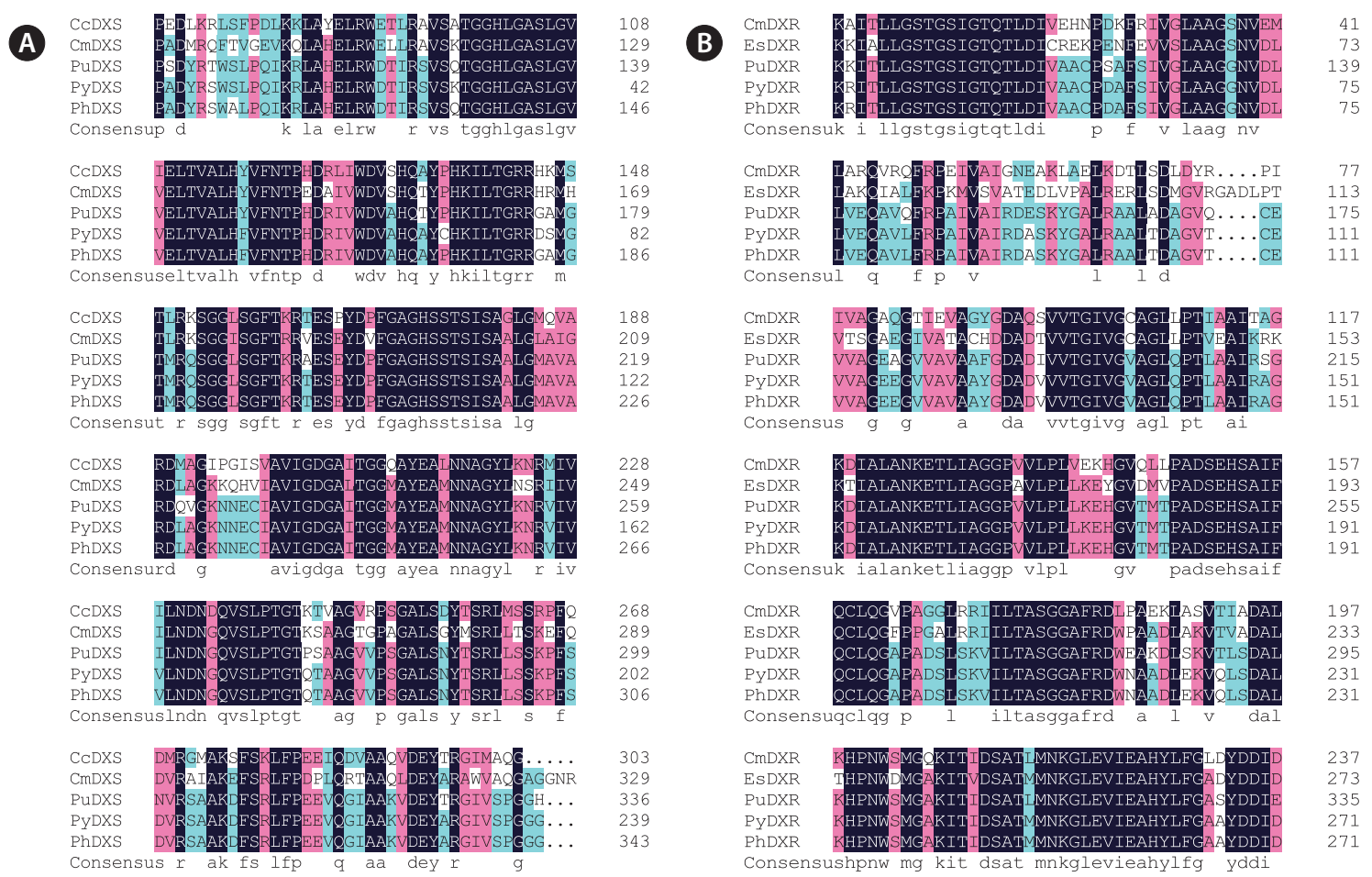

268
289
299
202
306
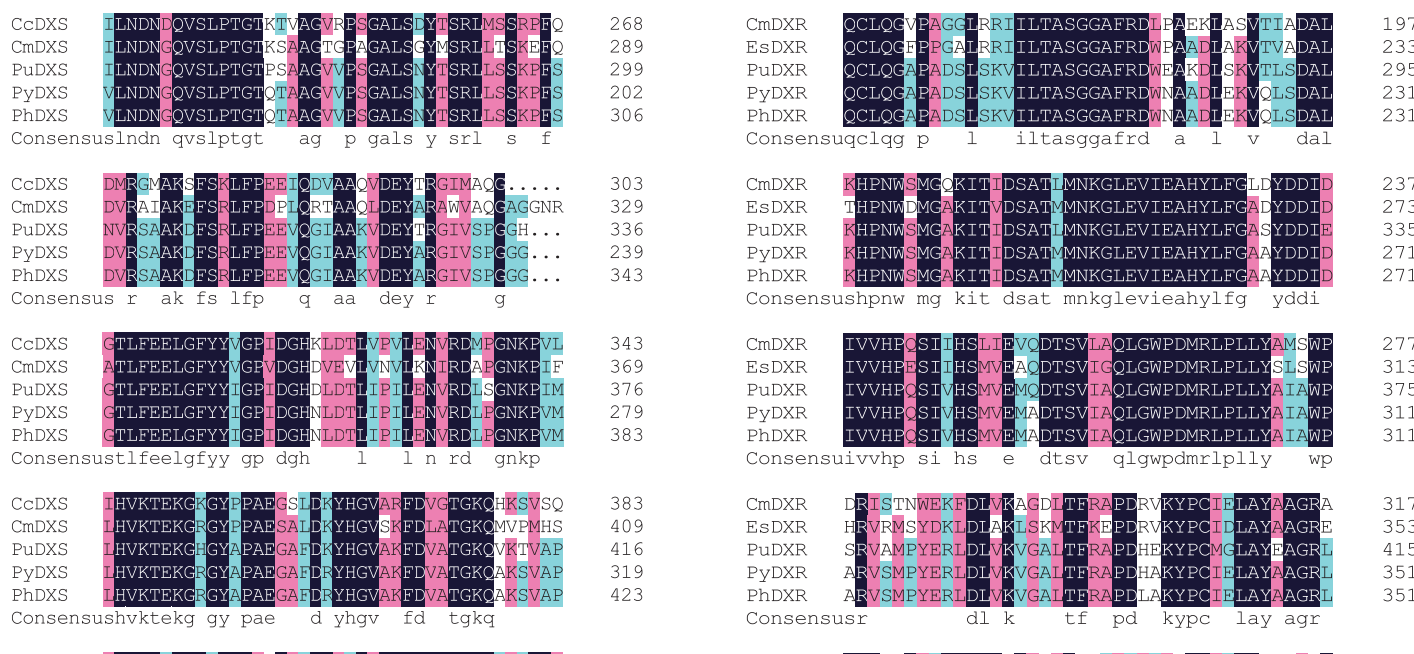

343
369
376
279
383

CMDXR
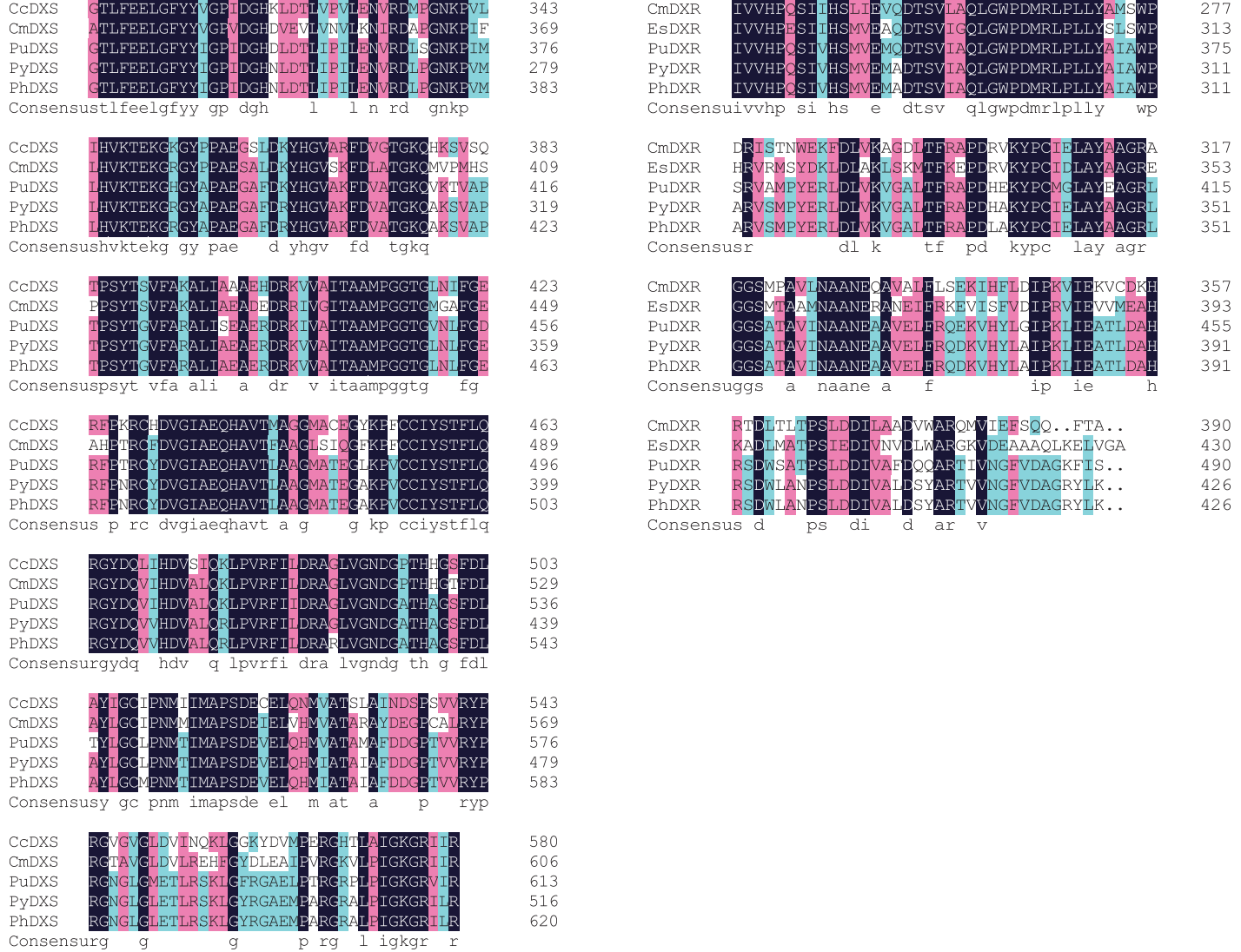

Fig. 5. Alignment of deduced amino acid sequences for DXS and DXR from different species. (A) PhDXS with other known DXS from Chondrus crispus (CcDXS, XP_005716785), Cyanidioschyzon merolae strain 10D (CmDXS, XP_005535753), Pyropia umbilicalis (PuDXS, esi sotig02238), and Pyropia yezoensis (PyDXS, FJ175680). (B) PhDXR with other known DXR from Chamaesiphon minutus (CmDXR, WP_015160708), Ectocarpus siliculosus (EsDXR, CBJ29720), Pyropia umbilicalis (PuDXR, esContig5003), and Pyropia yezoensis (PyDXR, ACI45960). DXS, 1-deoxy-D-xylulose 5-phosphate synthase; DXR, 1-deoxy-D-xylulose 5-phosphate reductoisomerase; PhDXS, P. haitanensis DXS; PhDXR, P. haitanensis DXR. 
1 ATCCCACCTCCGTATTGCATCCCTCTCCCCCTCCCCCTACCTTCCCCTGCCCCCCTTCCCCCATCCCTCGGCTG

76 TTTCCGGTCCCTGCGGCAGTGGCAGCCATGGCGCCGTGCACCCGCGCGGCGTTTGCCGCGTCGCCGGCCTCGCTG

151

17

226

42

301

67

376

92

451

117

526

142

601

167

676

192

751

217

826

242

901

267

976

292

1051

317

1126

342

1201

367

1276

392

1351

417

1426

442

1501

467

1576

492

1651

517

1726

542

1801

567

1876

592

1951

617

2026

642

2101

667

2176

692

2251

717

2326

742

2401

2476

2551

2626

2701

2776

2851 \begin{tabular}{llllllllllllllll}
$M$ & A & P & C & $T$ & $R$ & A & A & F & A & A & S & P & A & S & $L$ \\
\hline
\end{tabular}

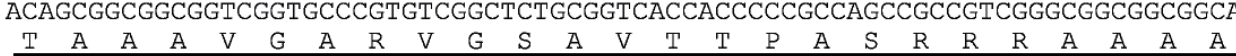
CCGCCGGATGGGGCCGCCTCGCGGCCCGCGGCGGTGCGCATGAACGCGCGTGGCGGCAATGGGGCGGTCCCGCCG

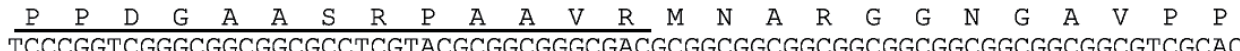

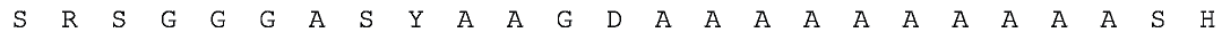
GACAATGGGCCCAAGAGCACCCCGCTGCTGGACCGGATTGCCGAGCCCGCCGACTACCGCAGCTGGGCGCTGCCG $\begin{array}{llllllllllllllllllllllllll}D & N & G & P & K & S & T & P & L & L & D & R & I & A & E & P & A & D & Y & R & S & W & A & L & P\end{array}$ CAGATCAAGCGGCTCGCGCACGAGCTCCGCTGGGACACCATCCGGTCCGTCTCGCAGACGGGCGGCCACTTGGGC $\begin{array}{lllllllllllllllllllllllll}Q & I & K & R & L & A & H & E & L & R & W & D & T & I & R & S & V & S & Q & T & G & G & H & L & G\end{array}$ GCCTCGCTAGGCGTCGTTGAGCTGACCGTCGCCCTCCACTTTGTGTTCAACACGCCCCACGACCGGATTGTGTGG $\begin{array}{ccccccccccccccccccccccccc}A & S & L & G & V & V & E & L & T & V & A & L & H & F & V & F & N & T & P & H & D & R & I & V & W \\ \end{array}$ GACGTGGCGCACCAGGCATACCCGCACAAGATCCTGACGGGCCGCCGTGGAGCGATGGGCACGATGCGGCAGAGC

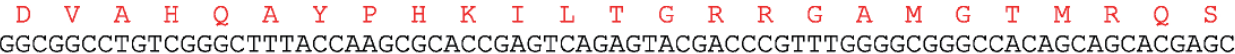
$\begin{array}{llllllllllllllllllllllllllll}G & G & L & S & G & F & T & K & R & T & E & S & E & Y & D & P & F & G & A & G & H & S & S & T & S\end{array}$ ATCTCGGCGGCGCTGGGCATGGCGGTCGCGCGGGACCTGGCGGGCAAGAACAATGAGTGCATTGCGGTGATTGGG $\begin{array}{llllllllllllllllllllllllll}I & S & A & A & L & G & M & A & V & A & R & D & L & A & G & K & N & N & E & C & I & A & V & I & G\end{array}$ GACGGCGCCATTACGGGTGGCATGGCATACGAGGCGATGAACAACGCGGGGTACCTGAAGAACCGGGTGATAGTG $\begin{array}{llllllllllllllllllllllllllllll}D & G & A & I & T & G & G & M & A & Y & E & A & M & N & N & A & G & Y & I & K & N & R & V & I & V \\ \end{array}$ GTGCTCAATGACAATGGGCAGGTGAGCCTACCGACGGGCACCCAGACGGCTGCGGGGGTGGTGCCGTCGGGGGCG $\begin{array}{lllllllllllllllllllllllllll}V & L & N & D & N & G & \& & V & S & L & P & T & G & T & \& & T & A & A & G & V & V & P & S & G & A\end{array}$ CTGTCCAACTACACCAGCCGGCTGCTGTCGTCCAAGCCCTTTTCCGACGTCCGGTCGGCGGCCAAGGACTTTTCG $\begin{array}{llllllllllllllllllllllllll}L & S & N & Y & T & S & R & L & L & S & S & K & P & F & S & D & V & R & S & A & A & K & D & F & S\end{array}$ CGCCTCTTCCCCGAGGAGGTGCAGGGCATTGCCGCCAAGGTGGACGAGTACGCGCGGGGTATTGTGTCGCCCGGC $\begin{array}{lllllllllllllllllllllllll}R & L & F & P & E & E & V & Q & G & I & A & A & K & V & D & E & Y & A & R & G & I & V & S & P & G\end{array}$ GGCGGGGGCACCCTGTTTGAGGAGCTGGGCTTTTACTACATTGGCCCCATTGACGGGCACAACCTGGACACGCTC $\begin{array}{ccccccccccccccccccccccccc}G & G & G & T & L & F & E & E & L & G & F & Y & Y & I & G & P & I & D & G & H & N & L & D & T & L\end{array}$ $\begin{array}{lllllllllllllllllllllllllll}I & P & I & L & E & N & V & R & D & L & P & G & N & K & P & V & M & L & H & V & K & T & E & K & G\end{array}$ CGCGGCTACGCCCCCGCCGAGGGCGCCTTTGACCGGTACCACGGGGTGGCCAAGTTTGACGTTGCCACTGGCAAG $\begin{array}{lllllllllllllllllllllllllll}R & G & Y & A & P & A & E & G & A & F & D & R & Y & H & G & V & A & K & F & D & V & A & T & G & K\end{array}$ CAGGCCAAGTCGGTGGCGCCGACGCCCTCTTACACGGGCGTCTTTGCCCGGGCACTCATTGCCGAGGCGGAGCGG $\begin{array}{llllllllllllllllllllllllllll}Q & A & K & S & V & A & P & T & P & S & Y & T & G & V & F & A & R & A & \text { L } & \text { I } & \text { A } & \text { E } & \text { A } & \text { E } & \text { R }\end{array}$ GACCGCAAGGTGGTGGCCATCACCGCGGCCATGCCGGGTGGGACGGGCCTCAACCTGTTTGGTGAGCGCTTCCCC

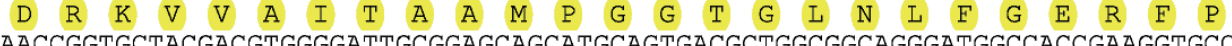
$\begin{array}{llllllllllllllllllllllllllll}N & R & C & Y & D & V & G & I & A & E & Q & H & A & V & T & L & A & A & G & M & A & T & E & G & A\end{array}$ AAGCCCGTCTGCTGCATTTACTCGACCTTCCTCCAGCGCGGCTATGACCAGGTCGTGCACGATGTGGCGCTGCAG

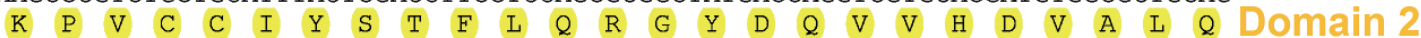
CGGCTACCGGTCCGCTTCATCCTCGACCGGGCGAGGCTTGTTGGCAACGATGGCGCTACTCATGCCGGGTCGTTT $\begin{array}{llllllllllllllllllllllllllllll}R & L & P & V & R & F & I & L & D & R & A & R & L & V & G & N & D & G & A & T & H & A & G & S & F\end{array}$ GACCTGGCCTACCTGGGCTGCATGCCCAACATGACCATCATGGCGCCGTCGGACGAGGTGGAGCTCCAGCACATG $\begin{array}{lllllllllllllllllllllllllll}\text { D } & \text { L } & \text { A } & \text { Y } & \text { L } & \text { G } & \text { C } & \text { M } & \text { P } & \text { N } & \text { M } & \text { T } & \text { I } & \text { M } & \text { A } & \text { P } & \text { S } & \text { D } & \text { E } & \text { V } & \text { E } & \text { L } & \text { Q } & \text { H } & \text { M }\end{array}$ ATCGCCACGGCCATTGCGTTTGATGATGGCCCGACGGTGGTGCGGTACCCTCGAGGCAACGGTCTCGGCCTCGAG $\begin{array}{llllllllllllllllllllllllllll}I & A & T & A & I & A & F & D & D & G & P & T & V & V & R & Y & P & R & G & N & G & L & G & L & E\end{array}$ ACGCTCCGGTCCAAGCTTGGCTACCGCGGCGCTGAGATGCCGGCGCGCGGGCGGGCGCTGCCTATTGGCAAGGGT $\begin{array}{llllllllllllllllllllllllll}T & L & R & S & K & L & G & Y & R & G & A & E & M & P & A & R & G & R & A & L & P & I & G & K & G\end{array}$ CGCATCCTCCGCCACGCCTCCGGCAATGGCGGGCGTAAGGTGGCGCTCCTGTCGATTGGCACGCGGCTGCTCGAG $\begin{array}{lllllllllllllllllllllllll}R & I & L & R & H & A & S & G & N & G & G & R & K & V & A & L & L & S & I & G & T & R & L & L & E\end{array}$ TGCGTCAATGCGGCAGACGCGCTCGAGGCGGCAGGCGTGGCCGTGACCGTTGCGGACGCACGGTTCATGAAGCCT $\begin{array}{llllllllllllllllllllllllll}C & \text { V } & N & A & A & D & A & I & E & A & A & G & V & A & V & T & V & A & D & A & R & F & M & K & P & \end{array}$ GACAGTGGAGGAGGGCAGCATTGGG $\begin{array}{lllllllllllllllllllllllll}\text { L } & \text { D } & \text { T } & \text { E } & \text { L } & \text { V } & \text { R } & \text { S } & \text { L } & \text { A } & \text { A } & \text { D } & \text { H } & \text { E } & \text { V } & \text { L } & \text { V } & \text { T } & \text { V } & \text { E } & \text { E } & \text { G } & \text { S } & \text { I } & G \\ \text { GGCTTTGGCGACCATGTGCTCCACTTTCTGGCCCTCGAGGGGCTCCTCGACGGTGGTGACCTGCGGGTGCGGCCG }\end{array}$ $\begin{array}{lllllllllllllllllllllllllll}G & F & G & D & H & V & L & H & F & L & A & L & E & G & L & L & D & G & G & D & L & R & V & R & P\end{array}$ ATGGTCCTCCCCGACCGGTACATTGACCACGGGAGCCAGAGCGAGCAGTACGAGGAGGCGGGGCTGTATGCGAGC $\begin{array}{llllllllllllllllllllllllllllllllll}M & V & L & P & D & R & Y & I & D & H & G & S & Q & S & E & Q & Y & E & E & A & G & I & Y & A & S\end{array}$ CACATTGAGGCGACGGCCCTGCGCCTGCTGGGCCGCAGCGCAGAAGTGCTGTCGGGAGGCGTCAGCTCTTGAGCC $\begin{array}{lllllllllllllllllllllll}H & I & E & A & \text { T } & A & \text { L } & R & \text { L } & \text { L } & G & R & S & A & E & V & L & S & G & G & V & S & S\end{array}$ GGCGACCGTTGCGTCCGTGGCGGGCGGACGGGTCTTGGGCCTCGCATCACCTTCCCATTCTTCCTTTCGTCTTTT TTTCTTCTGAGAGCCGCTTGGCGACTGCTCTTTTACTCTTCCTGCGTACCGTAATGCCGCTGGCGGCGGCCACCG CCGGTCCGTGGTCAGCTGCTGCCAGGCCTGGTCGGTGGGCCGGGCGAGCCTACCAATGCCCCTCGGGTTCATTGT CCCCGCTGCTGTACTTTGTGCGGCTGTGCCTGCCGATGTGGCTGATGAGAGCACGACTTGGCCGCCCGAGCCGCA GAGGCTTGTCGTCGGTCGGGCGTCCTCCCCCCGCGTGCCGGCCGTTGCTGTACAGTGCATACAGTGTACATACTG TACCGATGCCTGTGGATTCCCCCCGTGGTGCCCGCCGTGCGTCCGGTCCTGTGTGTGCCGTGGCCTCCGTCGCGC CGCTGACGTGGCCGGGAGTGGCGGCTGTGGCCGCCAACCGTTTTCTGACCAATCGTCGTGG 2911

Fig. 6. The CDNA and deduced amino acid sequences of DXS from Pyropia haitanensis. The start codon (ATG) and stop codon (TGA) are in bold; domain 1 is in red, domain 2 is in yellow, domain 3 is in blue; transit peiptides are underlined by black line. DXS, 1-deoxy-D-xylulose 5-phosphate synthase. 


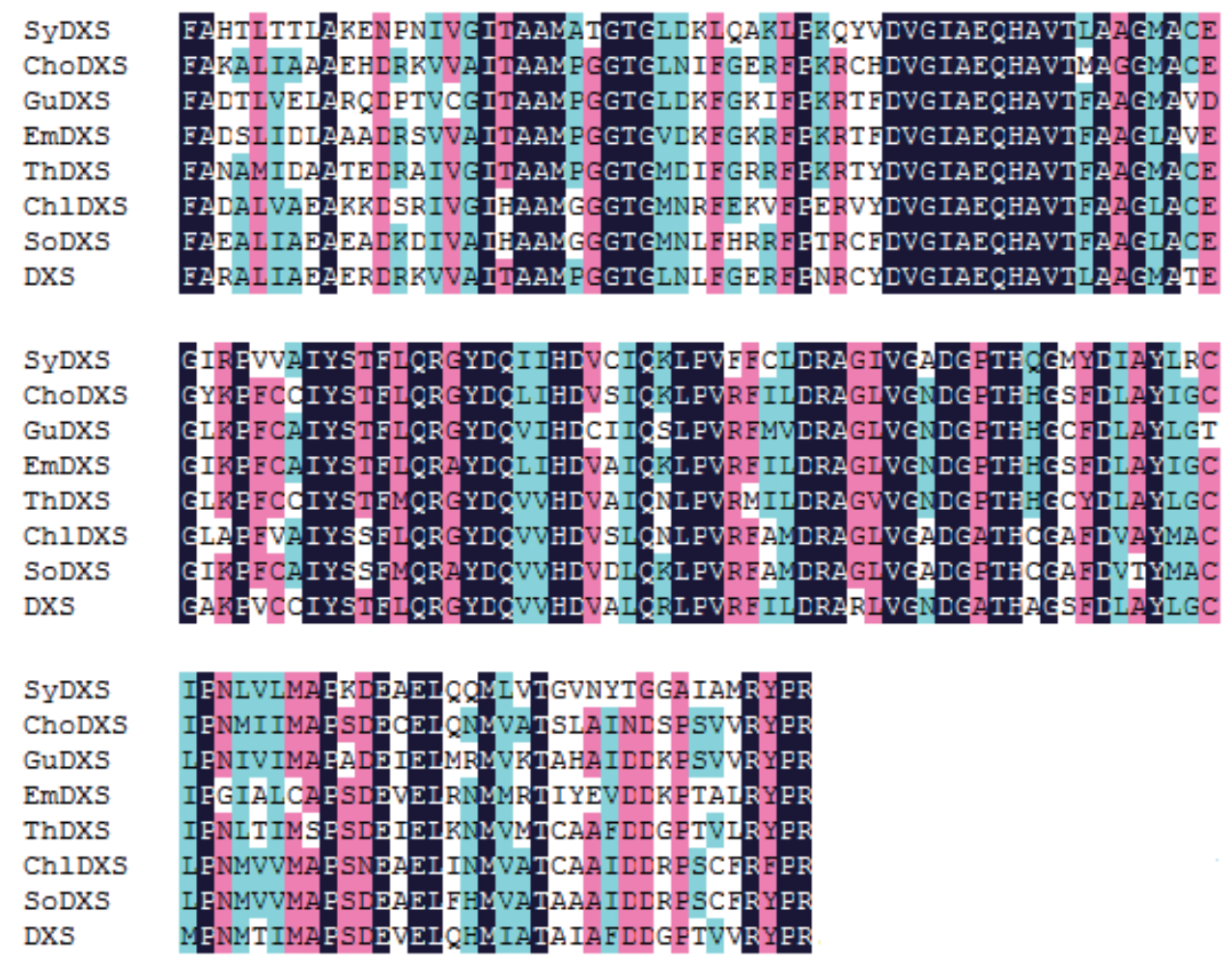

Fig. 7. Multiple alignments of DXS TPP_PYR_DXS_TK_like amino acid sequences from different species (PhDXS in Pyropia haitanensis; SyDXS in Synechocystis sp. PCC6803, BAK49262.1; ChoDXS in Chondrus crispus, XP_005716785.1; GuDXS in Guillardia theta CCMP2712, EKX37283.1; EmDXS in Emiliania huxleyi CCMP1516, EOD35929.1; ThDXS in Thalassiosira pseudonana CCMP1335, ACI64314.1; ChIDXS in Chlorella variabilis, EFN58715.1; SoDXS in Solanum lycopersicum, NP_001234553.2).

bp at the 5' UTR and 3' UTR. The ORF of these two genes $P h D X S$ and $P h D X R$ encoded predicted polypeptides of 764 and 426 amino acids residues, respectively. The deduced PhDXS protein had a theoretical pI of 7.71 and a molecular mass of $79.9 \mathrm{kDa}$ while $P h D X R$ protein was 5.07 at theoretical $\mathrm{pI}$ and $44.4 \mathrm{kDa}$ in the molecular weight.

On the amino acid level, alignment of PhDXS shared $74 \%$ sequence identity with DXS from Chondrus crispus, $69 \%$ similarity with Cyanidioschyzon merolae strain 10D DXS (Fig. 5A) and 100\% sequence identity with the Sequence Read Archive (SRA) database of Pyropia haitanensis from BioProjects PRJNA282903 (Wang et al. 2015), PRJNA181961 (Xie et al. 2013). Meanwhile, the deduced amino acid sequence of PhDXR displayed a certain number of homology with the DXR sequences (Fig. 5B) from Pyropia yezoensis (99\% identity), Chamaesiphon minutus (69\% identity), Ectocarpus siliculosus (63\% identity) and the big database of P. haitanensis from BioProjects PRJNA282903, PRJNA181961 (99\% identity). Through blastx analysis, results revealed that the amino acids sequence of DXS / DXR from different algae all contained a characteristic domain among DXS / DXR enzyme.

\section{Bioinformatic analysis of $P h D X S$ and $P h D X R$}

The encoding PhDXS protein predicted by NCBI Conserved Domain Search indicated that it contained three conservative domains (Fig. 6): domain I for DXP synthase_N (101-388), domain II for TPP_PYR_ DXS_ TK_like (430-584), and domain III for Transketolase_C (614-743). The three domains belonged to TPP_enzymes superfamily, TPP_enzymes_PYR superfamily, and Transketolase_C superfamily, respectively. Domain I contained one TPP-binding site while domain II possessed not only one TPP-binding site but also two peptide-binding regions of PYR / PP interface and dimer interface. Through multiple alignments of DXS domain II amino acid sequences from different species (Fig. 7), we came to a conclusion that DXS TPP_PYR_DXS_TK_like domain shared a high level of conservatism, especially at the amino acid residues of PYR / PP interface peptide-binding 

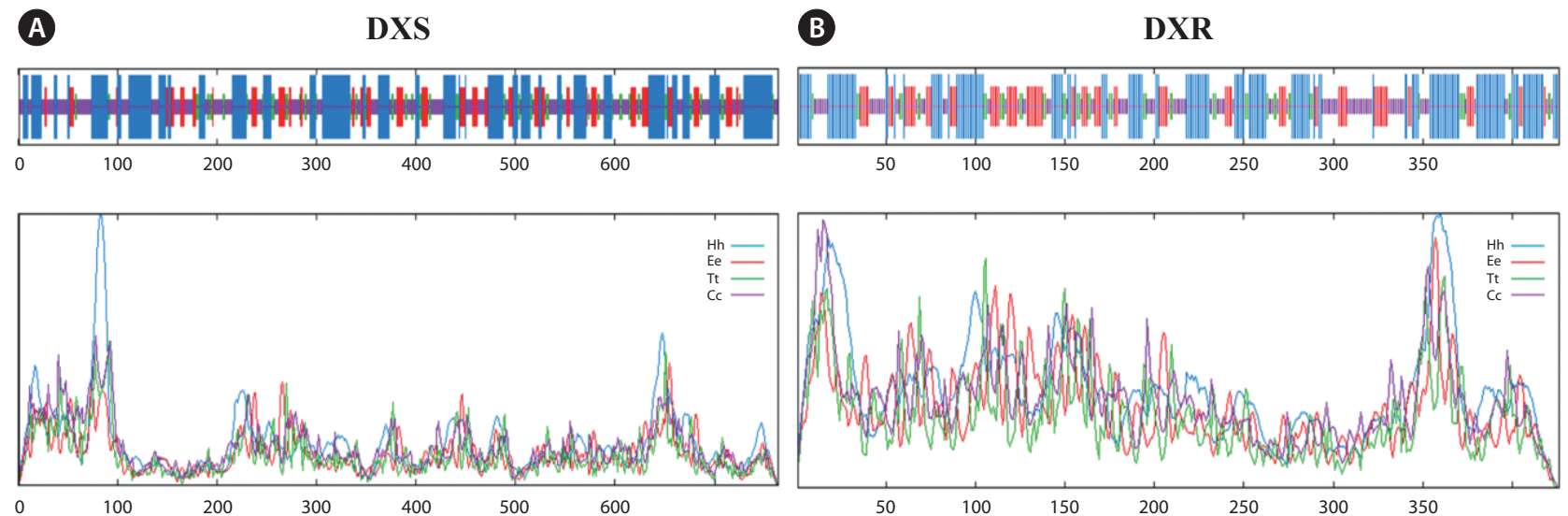

Fig. 8. SOPMA secondary structure prediction results of two proteins from Pyropia haitanensis. (A) DXS. (B) DXR. Hh, Ee, Tt, and Cc represent alpha helix, extended strand, beta turn, and random coil, respectively. DXS, 1-deoxy-D-xylulose 5-phosphate synthase; DXR, 1-deoxy-D-xylulose 5-phosphate reductoisomerase.

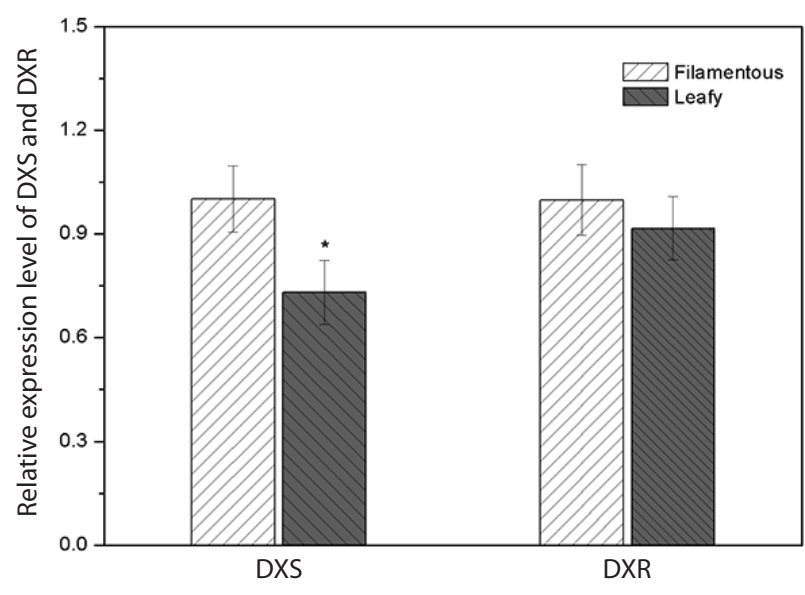

Fig. 9. Relative expression levels of DXS and DXR in different life history from Pyropia haitanensis. DXS, 1-deoxy-D-xylulose 5-phosphate synthase; DXR, 1-deoxy-D-xylulose 5-phosphate reductoisomerase. Each value is the mean \pm standard error of 3 independent biological replicates, the significance level was set at alpha $=0.05\left(^{*}\right)$.

site within plants.

The prediction of transmembrane structure by TMHMM Server v. 2.0 showed that $P h D X S$ didn't exist a transmembrane structure. DXS was known as a nuclear gene encoding protein in higher plants, and then located in the cell plastid through particular transport way (Xu et al. 2014). Therefore, TargetP 1.1 Server was used to indicate that PhDXS ultimately located in plastid rather than endoplasmic reticulum, mitochondria and other subcellular structures. By SignalP 4.1 Server and ChloroP 1.1 Server prediction, we confirmed that there was inexistence of a signal peptide whereas existence of a transit peptide with a length of 54 bp in PhDXS. Based on NPS@ SOPMA, The secondary structure of $P h D X S$ showed that it was a stabilizing protein rich in alpha helix (Fig. 8A), which approximately consisted of $\alpha$-helices (38.87\%), $\beta$-turns (10.08\%), extended strands (15.84\%), and random coils (35.21\%).

The PhDXR contained three conservative domains: domain I for DXP_reductoisom, domain II for DXP redisom_C and domain III for DXPR_C. The three domains belonged to NADB_Rossmann superfamily, DXP_redisom_C superfamily and DXPR_C superfamily, respectively. Targetscans showed that $P h D X R$ didn't exist a transmembrane structure, but existed a transit peptide with 18 bp in length. Like DXS, DXR was also known as a nuclear gene encoding protein which located in the cell plastid (Hans et al. 2004). The secondary structure of $P h D X R$ showed that it was a stabilizing protein rich in alpha helix (Fig. 8B), which mainly consisted of an alpha helix of $40.85 \%$, a beta turn of $11.03 \%$, a extended strand of $20.89 \%$, and a coil of $27.23 \%$.

\section{Expression analysis of PhDXS and PhDXR in dif- ferent life history}

Expression of $P h D X S$ and $P h D X R$ were carried in heteromorphic life cycles between leafy thallus and filamentous conchocelis by analysis of real-time quantitative PCR. We found that expression of PhDXS as well as $P h D X R$ was higher in conchocelis as compared with leafy thallus, that expression quantity of $P h D X S$ and $P h D X R$ in leafy thallus were $0.728 \pm 0.185$ and $0.913 \pm 0.204$ fold as compared to them in conchocelis (Fig. 9). Observably higher accumulation of PhDXS was surveyed in concho- 
celis whereas no significant difference was surveyed for $P h D X R$ in conchocelis when it was compared to leafy thallus. Semiquantitative RT-PCR analysis proved this conclusion as well (Fig. 10). DXS and DXR both participated in regulation of carbon flow whereas playing different roles among the MEP pathway. By relative expression analysis of DXR / DXS (Fig. 11), we found that expression quantity of DXR was $106.87 \pm 9.7$ fold as compared to DXS in leafy thallus as DXR being $134.08 \pm 11.0$ fold as compared to DXS in conchocelis. No significant change in expression of DXR / DXS suggested that the effect of $P h D X S$ and PhDXR among the MEP pathway makes no difference in different life history.

\section{Expression of GGPS in conchocelis of Pyropia haitanensis under fosmidomycin treatment}

Through the comparative analysis of GGPS transcripts of P. umbilicalis (P_umbilicalis_esContig5139) and GGPS of different species, The length of $612 \mathrm{bp}$ core fragment of downstream gene GGPS designed by primers GGPSF / GGPSR (Genbank accession No. KY697781) was obtained (Fig. 4A). In addition, by utilizing genomic DNA as a template and GGPSF / GGPSR as primers for amplification of GGPS, we concluded that there is no intron among PhGGPS. Through alignment analysis, this part of the coded amino acid had $95 \%$ and $66 \%$ identity to the GGPS from P. umbilicalis (AMA76411) and Emiliania huxleyi CCMP1516 (XP_005779411), 100\% identity to the big database of P. haitanensis from BioProjects PRJNA282903, PRJNA181961, respectively. The unique structure of multiple-domain PLN02890 in these three algal GGPS proteins showed high conservatism.

In this experiment, quantitative real time polymerase chain reaction method was used to detect the expression of PhDXR and downstream PhGGPS under fosmidomycin treatment. As leafy thallus of $P$. haitanensis cultivated in sodium salt marine under fosmidomycin concentrations of $200 \mu \mathrm{M} \mathrm{L}^{-1}$ for $72 \mathrm{~h}$, concentrations of chlorophyll and phycobiliproteins from $P$. haitanensis were displayed in Table 2, we could find that compared with the control group, concentrations of chlorophyll, total carotenoids and phycobiliproteins under fosmidomycin concentrations of $200 \mu \mathrm{M} \mathrm{L}^{-1}$ significantly declined, which proving the inhibiting effect of fosmidomycin in the process of MEP pathway from P. haitanensis. Moreover, as filamentous conchocelis cultivated under fosmidomycin concentrations of 200 and $300 \mu \mathrm{M} \mathrm{L}^{-1}$ for $72 \mathrm{~h}$, expression quantity of DXR were $0.629 \pm 0.096$ and $0.309 \pm 0.115$ fold while GGPS been $0.632 \pm 0.044$ and $0.059 \pm 0.012$

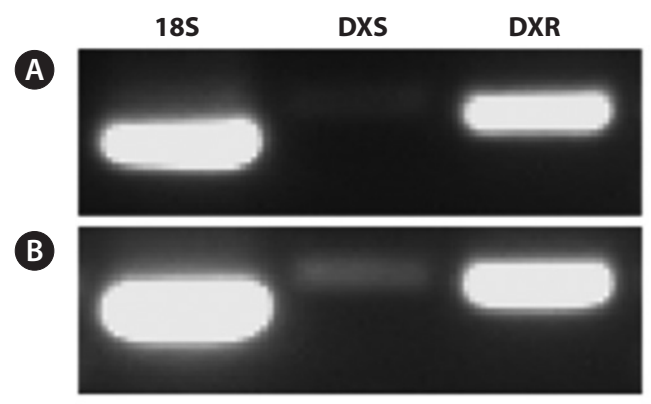

Fig. 10. The semi-quantitative PCR products of DXS and DXR from Pyropia haitanensis. (A) The leafy thallus. (B) The filamentous thallus. PCR, polymerase chain reaction; DXS, 1-deoxy-D-xylulose 5-phosphate synthase; DXR, 1-deoxy-D-xylulose 5-phosphate reductoisomerase.

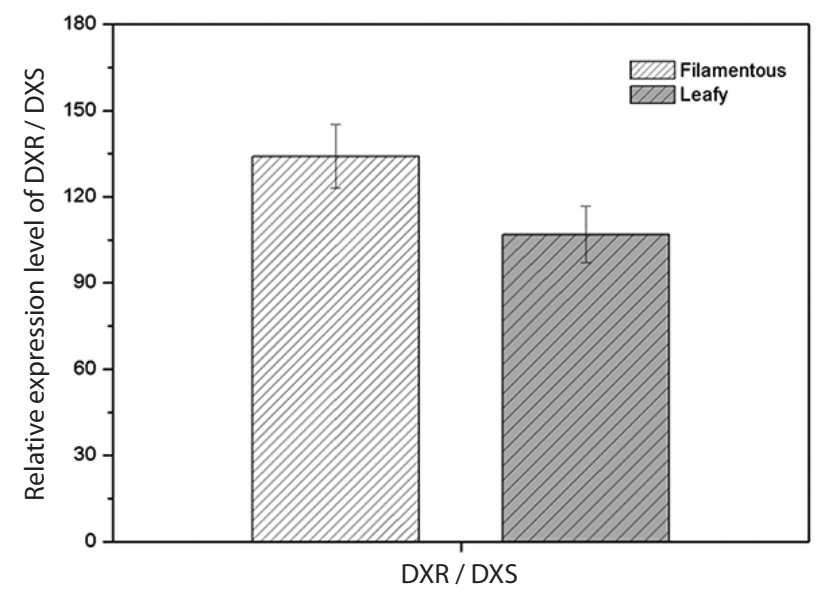

Fig. 11. Relative expression levels of DXR / DXS in different life history from Pyropia haitanensis. DXR, 1-deoxy-D-xylulose 5-phosphate reductoisomerase; DXS, 1-deoxy-D-xylulose 5-phosphate synthase. Each value is the mean \pm standard error of 3 independent biological replicates.

Table 2. Phytonutrients of Pyropia haitanensis under different concentrations of fosmidomycin

\begin{tabular}{|c|c|c|}
\hline Phytonutrients & $\begin{array}{c}\text { Control } \\
\left(0 \mu \mathrm{L} \mathrm{L}^{-1}\right)\end{array}$ & $\begin{array}{l}\text { Fosmidomycin } \\
\left(200 \mu \mathrm{M} \mathrm{L}^{-1}\right)\end{array}$ \\
\hline Chlorophyll & $19.83 \pm 0.15$ & $11.68 \pm 0.07^{\mathrm{a}}$ \\
\hline Total carotenoids & $6.36 \pm 0.04$ & $3.66 \pm 0.03^{\mathrm{a}}$ \\
\hline C-phycocyanin & $86.70 \pm 0.18$ & $68.24 \pm 0.14^{\mathrm{a}}$ \\
\hline Allophycocyanin & $31.99 \pm 0.14$ & $25.31 \pm 0.04^{\mathrm{a}}$ \\
\hline Phycobiliproteins & $118.69 \pm 0.28$ & $93.55 \pm 0.17^{\mathrm{a}}$ \\
\hline
\end{tabular}

Values are presented as means \pm SD $\left(\mathrm{mg} \mathrm{g}^{-1} \mathrm{DW}\right)(\mathrm{n}=3)$. ${ }^{\mathrm{a}}$ Significant differences $(p<0.05)$ when compared with the control. 


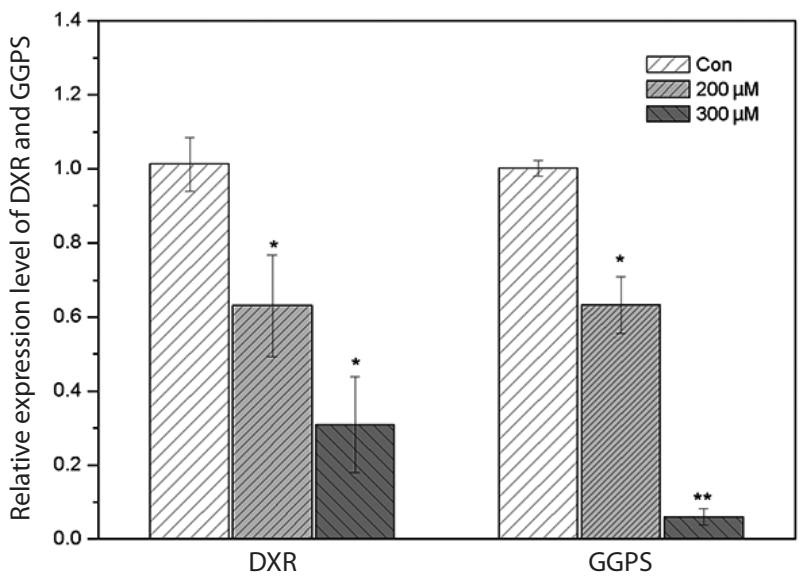

Fig. 12. Relative expression level of DXR and GGPS from Pyropia haitanensis after fosmidomycin treatment. DXR, 1-deoxy-D-xylulose 5-phosphate reductoisomerase; GGPS, geranylgeranyl diphosphate synthase. Each value is the mean \pm standard error of 3 independent biological replicates, the significance level was set at alpha $=0.01\left(^{* *}\right)$ / $0.05\left(^{*}\right)$.

fold as compared to the control groups with no fosmidomycin, respectively (Fig. 12). The results showed that there was significant decrease for expression of $P h D X R$ and $P h G G P S$ under fosmidomycin treatment, and more significant decrease with higher levels of fosmidomycin. Accordingly, we inferred that upstream genes like DXR among the MEP pathway can produce an influence on the expression of downstream gene GGPS for terpenoids biosynthesis.

\section{Phylogenetic analysis of PhDXS and PhDXR among algae DXSs and DXRs}

Phylogenetic tree was constructed using alignment of amino acid sequences of DXS and DXR from algae known as cyanobacteria, red algae, green algae, haptopflagellates, stramenopiles, dinoflagellate, and cryptophytes, to investigate the evolutionary relationship of PhDXS and PhDXR among the DXSs and DXRs of other algae. According to maximum likelihood phylogenetic tree based on DXS sequences (Fig. 13), we could see that it was totally divided into four classes, the first-level clustered into cyanobacteria and eukaryotes, as eukaryotes of the first-level clustering into green plants and Chromista. The second level of green plants clustered into Streptophyta plants and green algae, and Chromista clustered into red alga and its derivatives. Red algae derivatives of the third level clustered into the fourth level of haptopflagellates, cryptophytes, and stramenopiles. P. haitanensis was located at the third level of red algae, and shared closest relatives with $P$. yezoensis. The evolutionary tree nicely supported the "Cavalier-Smith endosymbiotic hypothesis." According to neighbor joining phylogenetic analysis on the basis of DXR sequences (Fig. 14), we came to a conclusion that $P$. haitanensis belonged to red algae and was closest to $P$. yezoensis in relatives. Besides, haptopflagellates, stramenopiles, and cryptophytes all evolved from endosymbiosis of red algae, which further supported the above mentioned theory. Frommolt et al. (2008) reported that the plastid of Dinoflagellates wasn't from red algae but from glaucophyte. Construction of PhDXR neighbor joining (NJ) phylogenetic tree exactly confirmed this viewpoint.

In higher plants, DXS protein was a typical multicore gene coding product (Peng et al. 2013). According to phylogenetic, gene expression, physiological and biochemical analysis of DXS, there were three types of DXS protein subtypes in higher plants (Xu et al. 2014, Davies et al. 2015), and these three protein subtypes had already been validated among angiosperms and gymnosperms (Cordoba et al. 2011). As a housekeeping gene, DXSI existed in green tissues to provide terpenoid synthetic precursor for the basic metabolism of photosynthesis and so on. DXSII and DXSIII genes existed in different tissues and subcellular units for selective expression (Carretero-Paulet et al. 2013, Tong et al. 2015). In the primary endosymbiotic green algae of Chlamydomonas reinhardtii, Volvox carteri, Ostreococcus tauri, Ostreococcus lucimarinus, etc., all the genes encoding enzymes in the MEP pathway were found to be single copies (Frommolt et al. 2008). Similarly, we only cloned a single PhDXS gene by RT-PCR and RACE, and the PhDXS protein was highly semblable with other red algae DXS proteins. According to NJ phylogenetic tree analysis based on DXS proteins (Fig. 15), the results showed that the genetic relationship of DXS proteins between P. haitanensis and Botryococcus braunii were similar to that of the DXSII protein subtype in higher plant. Xiang et al. (2007) reported that the function of DXSI protein in higher plant was most similar to that of the bacterial's among the three subtypes of the DXS protein. Meanwhile, according to the analysis from Fig. 14, we inferred the result as follows: Evolutionary sources of DXSI and DXSII in higher plants were not the same. 


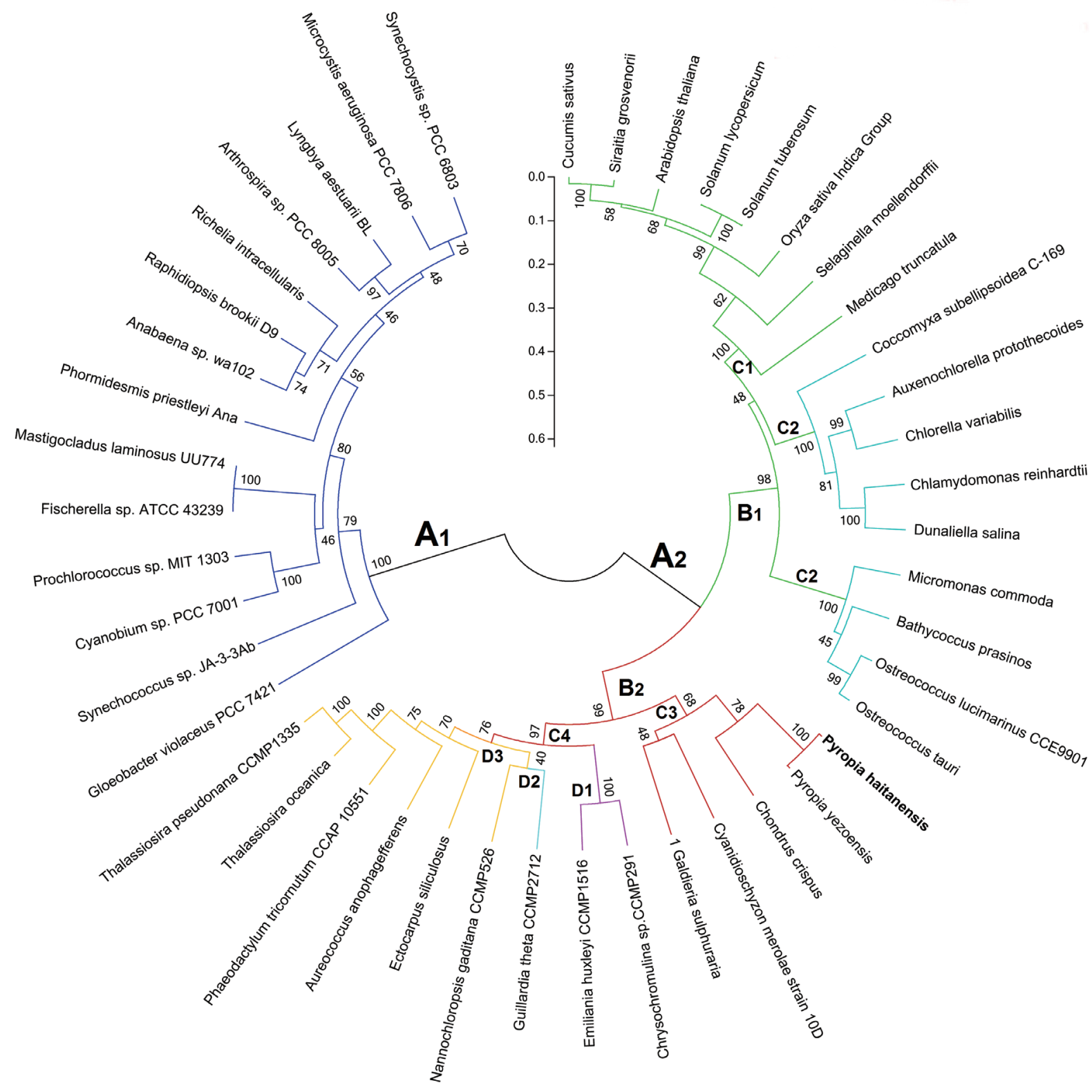

Fig. 13. Maximum likelihood phylogenetic analysis of translated full-length DXS sequence from Pyropia haitanensis. Amino acid sequences are aligned using the CLUSTAL W program, and phylogenetic tree using MEGA5 by poisson model. Bootstrap values obtained after 1,000 replications are shown on the branches. A1, prokaryotic cyanobacteria; A2, eukaryotic algae; B1, Viridiplantae; B2, Chromista; C1, Streptophyta; C2, green algae; C3, red algae; C4, red alga-like endosymbionts; D1, haptopflagellates; D2, cryptophytes; D3, stramenopiles. The accession numbers of the sequences are Anabaena sp. wa102 (ALB42737.1); Arabidopsis thaliana (AEE83625.1); Arthrospira sp. PCC8005 (CDM97627.1); Aureococcus anophagefferens (XP_009042014.1); Auxenochlorella protothecoides (XP_011397646.1); Bathycoccus prasinos (CCO19797.1); Chlamydomonas reinhardtii (EDO97255.1); Chlorella variabilis (EFN51764.1); Chondrus crispus (XP_005716785.1); Chrysochromulina sp. CCMP291 (KOO20858.1); Coccomyxa subellipsoidea C-169 (EIE22369.1); Cyanobium sp. PCC7001 (EDY39757.1); Cyanidioschyzon merolae strain 10D (BAM79467.1); Cucumis sativus (XP_004144970.1); Dunaliella salina (ACT21080.1); Ectocarpus siliculosus (CBJ28110.1); Emiliania huxleyi CCMP1516 (EOD35929.1); Fischerella sp. ATCC43239 (AIJ28521.1); Galdieria sulphuraria (EME28371.1); Gloeobacter violaceus PCC7421 (NP_923140.1); Guillardia theta CCMP2712 (EKX54585.1); Lyngbya aestuarii BL (ERT04218.1); Mastigocladus laminosus UU774 (KIY15048.1); Medicago truncatula (AES91764.1); Microcystis aeruginosa PCC7806 (CAO91355.1); Micromonas commoda (ACO68569.1); Nannochloropsis gaditana CCMP526 (XP_005854509.1); Oryza sativa Indica Group (EEC79215.1); Ostreococcus lucimarinus CCE9901 (ABO94483.1); Ostreococcus tauri (XP_003074992.1); Phormidesmis priestleyi Ana (KPQ35292.1); Phaeodactylum tricornutum CCAP10551 (EEC42622.1); Prochlorococcus sp. MIT1303 (KZR66594.1); Pyropia yezoensis (ACI45959.1); Raphidiopsis brookii D9 (EFA73351.1); Richelia intracellularis (CDN14922.1); Selaginella moellendorffii (EFJ33064.1); Siraitia grosvenorii (AEM42997.1); Solanum lycopersicum (NP_001234672.1); Solanum tuberosum (ADK73609.1); Synechococcus sp. JA-3-3Ab (ABC99857.1); Synechocystis sp. PCC6803 (BAK49262.1); Thalassiosira oceanica (EJK67229.1); Thalassiosira pseudonana CCMP1335 (XP_002295769.1). DXS, 1-deoxy-D-xylulose 5-phosphate synthase. 


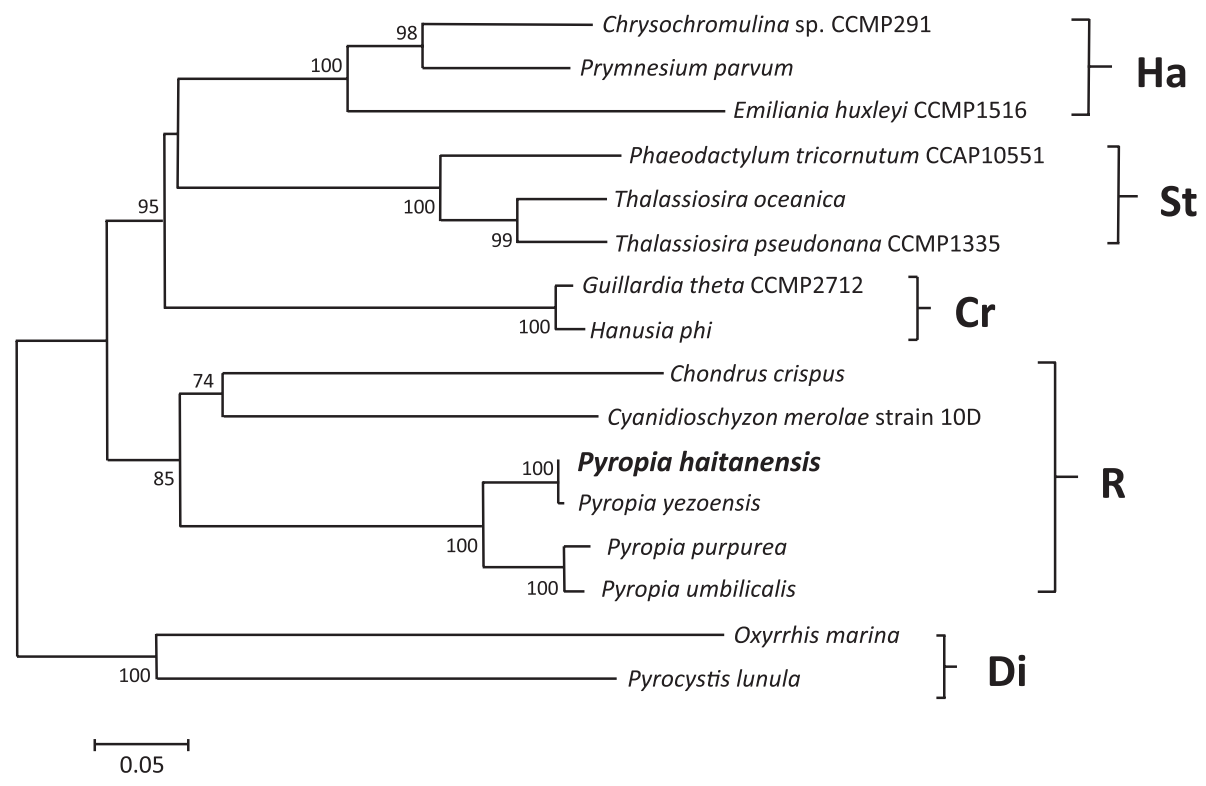

Fig. 14. Neighbor joining phylogenetic analysis of translated full-length DXR sequence from Pyropia haitanensis. The accession numbers of the sequences are Chondrus crispus (CDF41392.1); Chrysochromulina sp. CCMP291 (KOO25229.1); Cyanidioschyzon merolae strain 10D (BAM79654.1); Emiliania huxleyi CCMP1516 (EOD07466.1); Guillardia theta CCMP2712 (EKX37283.1); Hanusia phi (ABI96273.1); Oxyrrhis marina (ACE81815.1); Phaeodactylum tricornutum CCAP10551 (EEC51417.1); Prymnesium parvum (ABI96271.1); Pyrocystis lunula (ABI96272.1); Pyropia yezoensis (ACI45960.1); Thalassiosira oceanica (EJK49569.1); Thalassiosira pseudonana CCMP1335 (ACI64314.1). The sequence numbers of transcriptome are Pyropia purpurea (esi sotig05945); Pyropia umbilicalis (esContig5003). DXR, 1-deoxy-D-xylulose 5-phosphate reductoisomerase; Ha, haptopflagellates; St, stramenopiles; Cr, cryptophytes; R, red algae; Di, dinoflagellates.

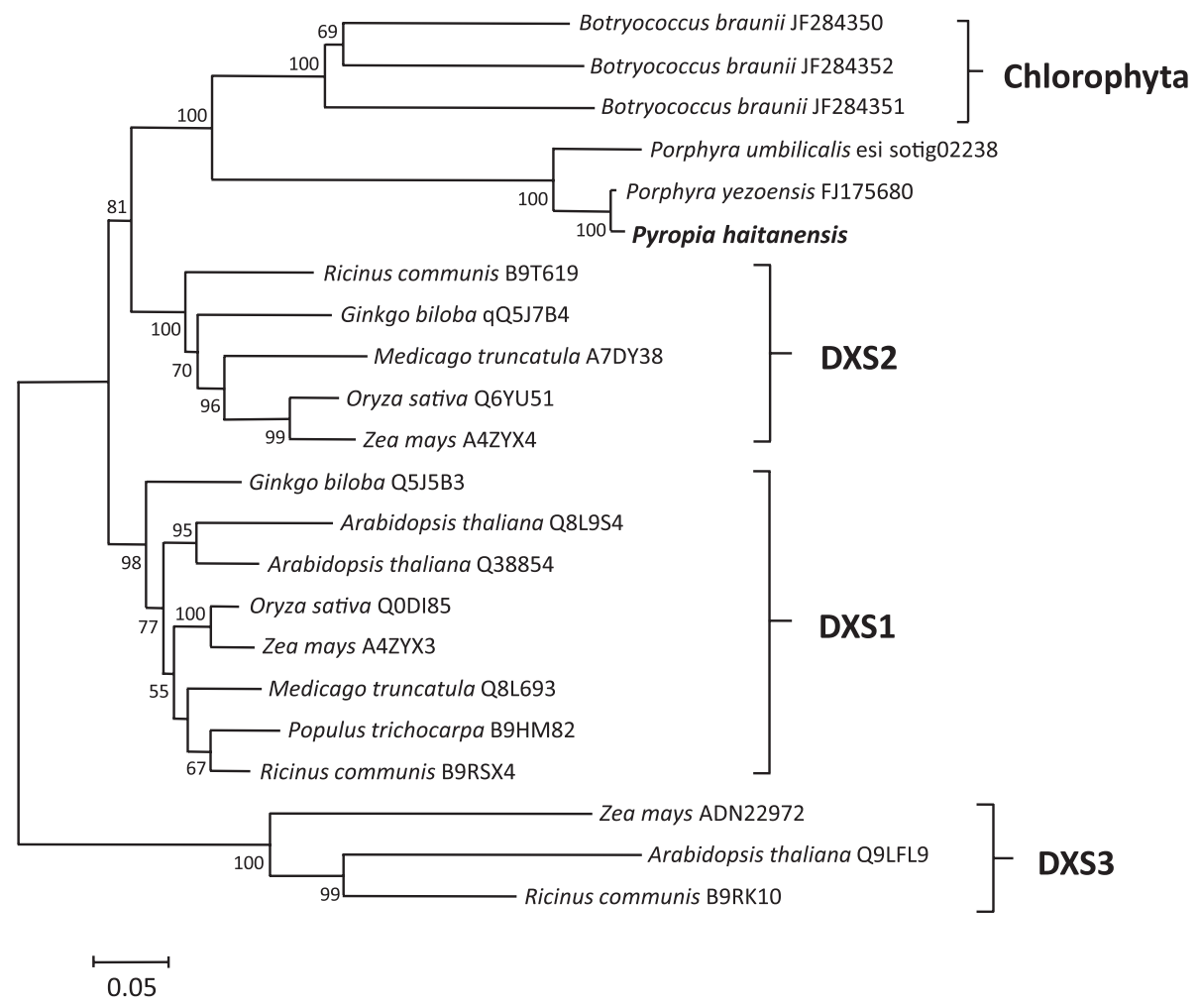

Fig. 15. Neighbor joining phylogenetic tree of DXS proteins from different organisms. The accession number of every DXS's is marked in the figure. DXS, 1-deoxy-D-xylulose 5-phosphate synthase. 


\section{DISCUSSION}

Terpenoids are the most widely distributed natural compounds in nature, playing an important role in agriculture, makeup and pharmaceutical industries (Davies et al. 2015). In recent one decade, though synthesis and metabolism research of terpenoid in higher plant has made great progress, red algae especially $P$. haitanensis terpene biosynthesis and metabolism has a lot of questions still unclear to date. Previous reports showed that high efficient synthesis of terpenoids would lead to the accumulation of biologically production and photosynthate during Rhodophyta formation (de Oliveira et al. 2012). In this study, we have cloned and characterized DXS and DXR from cDNA of $P$. haitanensis, which are, known as the first two rate-limiting genes in the MEP terpene synthesis pathway. Sequence alignments showed that the two genes contained no intron in their coding region. Bioinformatic analysis revealed that DXS and DXR proteins coded by nuclear genes are ultimately localized to the cytosome under the guidance of the transit peptide (DXS with 54 amino acid residues, DXR with 18 amino acid residues), which shows that proteins encoded by cDNA of PhDXS and PhDXR are functional and of great active.

Expression analysis of higher plant showed a huge difference between DXS and DXR in different growing periods (Tong et al. 2015). Xu et al. (2012) analyzed Porphyra yezoensis of different life cycles and suggested that photosynthetic performance and metabolic rate related to terpene biosynthesis in conchocelis phase were superior to that in leafy thallus phase. In addition, expression quantity of alternative oxidase (AOX) gene in Porphyra was also highly reflected in conchocelis phase, which proved that metabolic activity quite differentiated in different life history (Zhang et al. 2014). In our study, we have found that DXS and DXR cloned from P. haitanensis both expressed strongly in conchocelis of terpene synthesis, which was consistent with the above statements. We have also found that PhDXS had a more significant effect in the MEP pathway compared to $P h D X R$, but the effect of $P h D X S$ and $P h D X R$ made no difference in different life history. In addition, we investigated the issue whether expression levels of upstream and downstream genes among terpene biosynthesis in P. haitanensis are consistent. Fosmidomycin has been identified as a natural antibiotic inhibiting activity of the DXR enzyme and then preventing the conversion of DXP to MEP, consequently not only decreased activity of the downstream gene like GGPS but also interrupted the carotenogenesis in algae (Massé et al. 2004, Paniagua-Michel et al. 2009). Paniagua-Michel et al. (2009) also reported that at concentrations below $150 \mu \mathrm{M}$, fosmidomycin would have no influence on the carotenoid biosynthesis and the growth of algal cells. In our studies, we observed that PhDXR and PhGGPS were synchronized inhibited in fosmidomycin treatment, and more significant inhibition with higher levels of fosmidomycin above $200 \mu \mathrm{M}$.

Before genomic and transcriptome analysis of different algae, the basic biological evolutionary relationship between algae should be clarified (Lohr et al. 2012). According to the theory of endosymbiosis, plastids are formed by phagocytosis of single-celled organisms from cyanobacteria. In primary endosymbiosis, many cyanobacteria genes were transferred to the host nuclear genome, and then products of the gene coding proteins are repositioned to the cytosome (Reyes-Prieto et al. 2006). The first endosymbiosis forms three existing lineage, namely red algae, glaucophytes, green algae and chloroplastida (Adl et al. 2005). In secondary endosymbiosis, organisms with primary plastid such as red algae were phagocytosed again by other heterotrophic eukaryotic and formed the symbiont, it gradually formed secondary endosymbiotic organisms through the integration of genes (Lohr et al. 2012). Large amounts of data about the existing nuclear gene and plastid coding protein has been confirmed that the plastids among Haptopflagellates, Stramenopiles (including diatom, brown algae, Chrysophyceae, etc.), Dinoflagellates and Cryptophytes were obtained from red algae through secondary endosymbiosis (Frommolt et al. 2008). In this study, we constructed and analyzed evolutionary trees of different species of DXS and DXR. The results indicated that plastids of primary endosymbiotic red algae and green plants (green algae and Streptophyta) were derived from phagocytosing Cyanobacteria, and secondary endosymbiotic Stramenopiles, Haptopflagellates and Cryptomonas originated by phagocytosing red algae. This systematic analysis strongly supports the Cavalier-Smith endosymbiotic theory in chromalveolata.

Terpenoid synthesis can be divided into three stages, and the first stage is the production of terpenoid synthetic precursor IPP, and its isomer DMAPP. There exist two MVA and MEP pathways for metabolic of IPP and DMAPP in the nature. In primary endosymbiotic event, the MVA pathway of primary endosymbiosis is derived from the primary heterotrophic eukaryotes, which has been confirmed by substantial evidences (Lohr et al. 2012). However, there is highly controversial at the source of the terpenoid synthetic MVA pathway in secondary endosymbiotic event (Lohr et al. 2012). Based on the analysis 
of genome, transcriptome, EST, physiological and biochemical data of Haptopflagellates and Stramenopiles (Table 3), we find that both of them simultaneously exist two terpenoid synthetic MVA and MEP pathways. By red algal genome and transcriptome analysis (Table 3), Porphyra umbilicalis and Cyanidioschyzon merolae 10D contain only one part of the HMGS gene sequence, while genes for all following steps in the MVA pathway are absent (Matsuzaki et al. 2004, Chan et al. 2012, Lohr et al. 2012). Porphyra purpurea included three genes (HMGS, HMGR, and MDD) in the terpenoid synthetic MVA pathway (Chan et al. 2012). By transcriptome analysis of Laurencia dendroidea, it exists integrated steps in the MEP synthetic and metabolic gene network but has not found any presence of gene for the MVA pathway (de Oliveira et al. 2012). Both green algae and Streptophyta are the primary endosymbiotic products, however, the terpenoid synthetic MVA pathway was lost during the evolution of green algae, which existing only one pathway of the MEP (Lohr et al. 2012). Surprisingly, C. zofingiensis has not only the complete MEP terpenoid metabolic gene network, but also the first two genes encoding enzymes and a HMGS gene, while the other genes in the MVA pathway are missing (Huang et al. 2016). Finally, based on the above clustering results of phylogenetic tree and the ex- isting EST and genome data analysis, we can infer that the MVA terpene precursor synthesis pathway of red algae is absent in its evolution process, and it contains only the complete MEP pathway to meet the requirements of its own terpenoids synthesis. The MVA terpene precursor synthesis pathway of red algae derivative is not from the endosymbiotic red algae, while may be from two or more times endosymbiosis within the heterotrophic eukaryotic host.

In a word, in this study, we have firstly cloned and analyzed two key genes PhDXS and PhDXR in the MEP pathway for terpenoid biosynthesis from $P$. haitanensis. We have also researched the problem of chromalveolata biological evolution with PhDXS and PhDXR genes. Such studies would be of great importance for understanding the biosynthesis and metabolism of Pyropia, and could provide a new way to classify chromalveolata. With more in-depth explorations, function and characterization of DXS, DXR and other key genes among the MEP pathway in P. haitanensis would be investigated stage by stage. In addition, how to improve the expression of key genes in the MEP pathway in order to improve the synthesis of terpenoids in P. haitanensis will be of a great interest for further study.

Table 3. Distribution of the MVA and the MEP pathways in examples of different species from genome and transcriptome analysis

\begin{tabular}{|c|c|c|c|}
\hline \multirow{2}{*}{ Organisms } & \multicolumn{2}{|c|}{ Pathway } & \\
\hline & MVA & MEP & \\
\hline \multicolumn{4}{|l|}{ Stramenopiles } \\
\hline Phaeodactylum tricornutum & + & + & $\begin{array}{l}\text { Bowler et al. (2008) } \\
\text { Genome }\end{array}$ \\
\hline Aureococcus anophagefferens & + & + & Genome \\
\hline Ectocarpus & + & + & Cock et al. (2010) \\
\hline \multicolumn{4}{|l|}{ Haptopflagellates } \\
\hline Emiliania huxleyi & + & + & Genome \\
\hline \multicolumn{4}{|l|}{ Chlorophytes } \\
\hline Coccomyxa sp. C-169 & - & + & Genome \\
\hline Chlorella zofingiensis & - & + & Huang et al. (2016) \\
\hline Chlamydomonas reinhardtii & - & + & Genome \\
\hline \multicolumn{4}{|l|}{ Streptophytes } \\
\hline Mesostigma viride & + & + & Grauvogel and Petersen (2007) \\
\hline Arabidopsis & + & + & Vranová et al. (2013) \\
\hline \multicolumn{4}{|l|}{ Red aglae } \\
\hline Cyanidioschyzon merolae 10D & - & + & Grauvogel and Petersen (2007), Matsuzaki et al. (2004) \\
\hline Porphyra purpurea & - & + & Chan et al. (2012) \\
\hline Porphyra umbilicalis & - & + & Chan et al. (2012) \\
\hline Laurencia dendroidea & - & + & de Oliveira et al. (2012) \\
\hline
\end{tabular}

Genome from US DOE Joint Genome Institute (http://www.jgi.doe.gov/genome-projects) 134-141.

MVA, mevalonate; MEP, 2-C-methyl-D-erythritol 4-phosphate. 


\section{ACKNOWLEDGEMENTS}

This work was funded by the National Natural Science Foundation of China (no. 41276134). We are also grateful to Professor Shan Lu, Nanjing University, for the guidance of this article. We also thank the Jiangsu Research Institute of Laver for providing conchocelis of Pyropia haitanensis strains.

\section{REFERENCES}

Adl, S. M., Simpson, A. G., Farmer, M. A., Andersen, R. A., Anderson, O. R., Barta, J. R., Bowser, S. S., Brugerolle, G., Fensome, R. A., Fredericq, S., James, T. Y., Karpov, S., Kugrens, P., Krug, J., Lane, C. E., Lewis, L. A., Lodge, J., Lynn, D. H., Mann, D. G., McCourt, R. M., Mendoza, L., Moestrup, O., Mozley-Standridge, S. E., Nerad, T. A., Shearer, C. A., Smirnov, A. V., Spiegel, F. W. \& Taylor, M. F. 2005. The new higher level classification of eukaryotes with emphasis on the taxonomy of protists. J. Eukaryot. Microbiol. 52:399-451.

Bennett, A. \& Bogorad, L. 1973. Complementary chromatic adaptation in a filamentous blue-green alga. J. Cell. Biol. 58:1245-1257.

Blouin, N. A., Brodie, J. A., Grossman, A. C., Xu, P. \& Brawley, S. H. 2011. Porphyra: a marine crop shaped by stress. Trends. Plant. Sci. 16:29-37.

Bowler, C., Allen, A. E., Badger, J. H., Grimwood, J., Jabbari, K., Kuo, A., Maheswari, U., Martens, C., Maumus, F. \& Otillar, R. P. 2008. The phaeodactylum genome reveals the evolutionary history of diatom genomes. Nature 456:239-244.

Carretero-Paulet, L., Cairó, A., Botella-Pavía, P., Besumbes, O., Campos, N., Boronat, A. \& Rodríguez-Concepción, M. 2006. Enhanced flux through the methylerythritol 4-phosphate pathway in Arabidopsis plants overexpressing deoxyxylulose 5-phosphate reductoisomerase. Plant Mol. Biol. 62:683-695.

Carretero-Paulet, L., Cairó, A., Talavera, D., Saura, A., Imperial, S., Rodríguez-Concepción, M., Campos, N. \& Boronat, A. 2013. Functional and evolutionary analysis of DXL1, a non-essential gene encoding a 1-deoxy-D-xylulose 5-phosphate synthase like protein in Arabidopsis thaliana. Gene 524:40-53.

Cavalier-Smith, T. 1999. Principles of protein and lipid targeting in secondary symbiogenesis: euglenoid, dinoflagellate, and sporozoan plastid origins and the eukaryote family tree. J. Eukaryot. Microbiol. 46:347-366.

Chan, C. X., Blouin, N. A., Zhuang, Y., Zäuner, S., Prochnik, S.
E., Lindquist, E., Lin, S., Benning, C., Lohr, M., Yarish, C., Gantt, E., Grossman, A. R., Lu, S., Müller, K., Stiller, J. W., Brawley, S. H. \& Bhattacharya, D. 2012. Porphyra (Bangiophyceae) transcriptomes provide insights into red algal development and metabolism. J. Phycol. 48:13281342.

Chen, C., Dai, Z., Xu, Y., Ji, D. \& Xie, C. 2016. Cloning, expression, and characterization of carbonic anhydrase genes from Pyropia haitanensis (Bangiales, Rhodophyta). J. Appl. Phycol. 28:1403-1417.

Cock, J. M., Sterck, L., Rouzé, P., Scornet, D., Allen, A. E., Amoutzias, G., Anthouard, V., Artiguenave, F., Aury, J. M. \& Badger, J. H. 2010. The Ectocarpus genome and the independent evolution of multicellularity in brown algae. Nature 465:617-621.

Cordoba, E., Porta, H., Arroyo, A., San Román, C., Medina, L., Rodríguez-Concepción, M. \& León, P. 2011. Functional characterization of the three genes encoding 1-deoxyD-xylulose 5-phosphate synthase in maize. J. Exp. Bot. 62:2023-2038.

Davies, F. K., Jinkerson, R. E. \& Posewitz, M. C. 2015. Toward a photosynthetic microbial platform for terpenoid engineering. Photosynth. Res. 123:265-284.

de Oliveira, L. S., Gregoracci, G. B., Silva, G. G. Z., Salgado, L. T., Filho, G. A., Alves-Ferreira, M., Pereira, R. C. \& Thompson, F. L. 2012. Transcriptomic analysis of the red seaweed Laurencia dendroidea (Florideophyceae, Rhodophyta) and its microbiome. BMC Genomics 13:487.

Frommolt, R., Werner, S., Paulsen, H., Goss, R., Wilhelm, C., Zauner, S., Maier, U. G., Grossman, A. R., Bhattacharya, D. \& Lohr, M. 2008. Ancient recruitment by chromists of green algal genes encoding enzymes for carotenoid biosynthesis. Mol. Biol. Evol. 25:2653-2667.

Grauvogel, C. \& Petersen, J. 2007. Isoprenoid biosynthesis authenticates the classification of the green alga Mesostigma viride as an ancient streptophyte. Gene 396:125133.

Hans, J., Hause, B., Strack, D. \& Walter, M. H. 2004. Cloning, characterization, and immunolocalization of a mycorrhiza-inducible 1-deoxy-d-xylulose 5-phosphate reductoisomerase in arbuscule-containing cells of maize. Plant Physiol. 134:614-624.

Huang, W., Ye, J., Zhang, J., Lin, Y., He, M. \& Huang, J. 2016. Transcriptome analysis of Chlorella zofingiensis to identify genes and their expressions involved in astaxanthin and triacylglycerol biosynthesis. Algal Res. 17:236-243.

Kuzuyama, T., Shimizu, T., Takahashi, S. \& Seto, H. 1998. Fosmidomycin, a specific inhibitor of 1-deoxy-d-xylulose 5-phosphate reductoisomerase in the nonmevalonate pathway for terpenoid biosynthesis. Tetrahedron. Lett. 
39:7913-7916.

Liu, J., Xu, Y., Liang, L. \&Wei, J. 2015. Molecular cloning, characterization and expression analysis of the gene encoding 1-deoxy-D-xylulose 5-phosphate reductoisomerase from Aquilaria sinensis (Lour.) Gilg. J. Genet. 94:239-249.

Lohr, M., Schwender, J. \& Polle, J. E. W. 2012. Isoprenoid biosynthesis in eukaryotic phototrophs: a spotlight on algae. Plant Sci. 185-186:9-22.

Luo, Q., Zhu, Z., Zhu, Z., Yang, R., Qian, F., Chen, H. \& Yan, X. 2014. Different responses to heat shock stress revealed heteromorphic adaptation strategy of Pyropia haitanensis (Bangiales, Rhodophyta). PLoS ONE 9:e94354.

Massé, G., Belt, S. T., Rowland, S. J. \& Rohmer, M. 2004. Isoprenoid biosynthesis in the diatoms Rhizosolenia setigera (Brightwell) and Haslea ostrearia (Simonsen). Proc. Natl. Acad. Sci. U. S. A. 101:4413-4418.

Matsuzaki, M., Misumi, O., Shin-I, T., Maruyama, S., Takahara, M., Miyagishima, S. Y., Mori, T., Nishida, K., Yagisawa, F., Nishida, K., Yoshida, Y., Nishimura, Y., Nakao, S., Kobayashi, T., Momoyama, Y., Higashiyama, T., Minoda, A., Sano, M., Nomoto, H., Oishi, K., Hayashi, H., Ohta, F., Nishizaka, S., Haga, S., Miura, S., Morishita, T., Kabeya, Y., Terasawa, K., Suzuki, Y., Ishii, Y., Asakawa, S., Takano, H., Ohta, N., Kuroiwa, H., Tanaka, K., Shimizu, N., Sugano, S., Sato, N., Nozaki, H., Ogasawara, N., Kohara, Y. \& Kuroiwa, T. 2004. Genome sequence of the ultrasmall unicellular red alga Cyanidioschyzon merolae 10D. Nature 428:653-657.

Okada, K., Saito, T., Nakagawa, T., Kawamukai, M. \& Kamiya, Y. 2000. Five geranylgeranyl diphosphate synthases expressed in different organs are localized into three subcellular compartments in Arabidopsis. Plant Physiol. 122:1045-1056.

Paniagua-Michel, J., Capa-Robles, W., Olmos-Soto, J. \& Gutierrez-Millan, L. E. 2009. The carotenogenesis pathway via the isoprenoid- $\beta$-carotene interference approach in a new strain of Dunaliella salina isolated from Baja California Mexico. Mar. Drugs 7:45-56.

Pattanaik, B. \& Lindberg, P. 2015. Terpenoids and their biosynthesis in cyanobacteria. Life (Basel) 5:269-293.

Peng, G., Wang, C., Song, S., Fu, X., Azam, M., Grierson, D. \& $\mathrm{Xu}, \mathrm{C} .2013$. The role of 1-deoxy-d-xylulose-5-phosphate synthase and phytoene synthase gene family in citrus carotenoid accumulation. Plant Physiol. Biochem. 71:67-76.

Reyes-Prieto, A., Hackett, J. D., Soares, M. B., Bonaldo, M. F. \& Bhattacharya, D. 2006. Cyanobacterial contribution to algal nuclear genomes is primarily limited to plastid functions. Curr. Biol. 16:2320-2325.

Rodríguez-Concepción, M., Ahumada, I., Diez-Juez, E.,
Sauret-Güeto, S., Lois, L. M., Gallego, F., CarreteroPaulet, L., Campos, N. \& Boronat, A. 2001. 1-Deoxy-Dxylulose 5-phosphate reductoisomerase and plastid isoprenoid biosynthesis during tomato fruit ripening. Plant J. 27:213-222.

Scolnik, P. A. \& Bartley, G. E. 1994. Nucleotide sequence of an Arabidopsis cDNA for geranylgeranyl pyrophosphate synthase. Plant Physiol. 104:1469-1470.

Slamovits, C. H. \& Keeling, P. J. 2008. Plastid-derived genes in the nonphotosynthetic alveolate Oxyrrhis marina. Mol. Biol. Evol. 25:1297-1306.

Sutherland, J. E., Lindstrom, S. C., Nelson, W. A., Brodie, J., Lynch, M. D. J., Hwang, M. S., Choi, H. -G., Miyata, M., Kikuchi, N., Oliveira, M. C., Farr, T., Neefus, C., MolsMortensen, A., Milstein, D. \& Müller, K. M. 2011. A new look at an ancient order: generic revision of the Bangiales (Rhodophyta). J. Phycol. 47:1131-1151.

Tong, Y., Su, P., Zhao, Y., Zhang, M., Wang, X., Liu, Y., Zhang, X., Gao, W. \& Huang, L. 2015. Molecular cloning and characterization of DXS and DXR genes in the terpenoid biosynthetic pathway of Tripterygium wilfordii. Int. J. Mol. Sci. 16:25516-25535.

Vranová, E., Coman, D. \& Gruissem, W. 2013. Network analysis of the MVA and MEP pathways for isoprenoid synthesis. Annu. Rev. Plant. Biol. 64:665-700.

Wang, L., Mao, Y., Kong, F., Cao, M. \& Sun, P. 2015. Genomewide expression profiles of Pyropia haitanensis in response to osmotic stress by using deep sequencing technology. BMC Genomics 16:1012.

Wellburn, A. R. 1994. The spectral determination of chlorophylls $a$, and $b$, as well as total carotenoids, using various solvents with spectrophotometers of different resolution. J. Plant. Physiol. 144:307-313.

Xiang, S., Usunow, G., Lange, G., Busch, M. \& Tong, L. 2007. Crystal structure of 1-deoxy-d-xylulose 5-phosphate synthase, a crucial enzyme for isoprenoids biosynthesis. J. Biol. Chem. 282:2676-2682.

Xie, C., Li, B., Xu, Y., Ji, D. \& Chen, C. 2013. Characterization of the global transcriptome for Pyropia haitanensis (Bangiales, Rhodophyta) and development of cSSR markers. BMC Genomics 14:107.

Xu, D., Qiao, H., Zhu, J., Xu, P., Liang, C., Zhang, X., Ye, N. \& Yang, W. 2012. Assessment of photosynthetic performance of Porphyra yezoensis (Bangiales, Rhodophyta) in conchocelis phase. J. Phycol. 48:467-470.

Xu, Y., Liu, J., Liang, L., Yang, X., Zhang, Z., Gao, Z., Sui, C. \& Wei, J. 2014. Molecular cloning and characterization of three cDNAs encoding 1-deoxy-d-xylulose-5- phosphate synthase in Aquilaria sinensis (Lour.) Gilg. Plant Physiol. Biochem. 82:133-141. 
Yang, L. -E., Huang, X. -Q., Lu, Q. -Q., Zhu, J. -Y. \& Lu, S. 2016. Cloning and characterization of the geranylgeranyl diphosphate synthase (GGPS) responsible for carotenoid biosynthesis in Pyropia umbilicalis. J. Appl. Phycol. 28:671-678.

Yang, L. -E., Jin, Q. -P., Xiao, Y., Xu, P. \& Lu, S. 2013. Improved methods for basic molecular manipulation of the red alga Porphyra umbilicalis (Rhodophyta: Bangiales). J. Appl. Phycol. 25:245-252.

Zhang, B. Y., Zhu, D. L., Wang, G. C. \& Peng, G. 2014. Characterization of the $A O X$ gene and cyanide-resistant respiration in Pyropia haitanensis (Rhodophyta). J. Appl. Phycol. 26:2425-2433. 